\title{
Linear Dynamics of the Semi-geostrophic Equations in Eulerian Coordinates on $\mathbb{R}^{3}$
}

\author{
Stefania Lisai(i) and Mark Wilkinson \\ Communicated by M. Hieber
}

\begin{abstract}
We consider a class of steady solutions of the semi-geostrophic equations on $\mathbb{R}^{3}$ and derive the linearised dynamics around those solutions. The linear PDE which governs perturbations around those steady states is a transport equation featuring a pseudo-differential operator of order 0 . We study well-posedness of this equation in $L^{2}\left(\mathbb{R}^{3}, \mathbb{R}^{3}\right)$ introducing a representation formula for the solutions, and extend the result to the space of tempered distributions on $\mathbb{R}^{3}$. We investigate stability of the steady solutions of the semi-geostrophic equations by looking at plane wave solutions of the associated linearised problem, and discuss differences in the case of the quasi-geostrophic equations.
\end{abstract}

Mathematics Subject Classification. Primary 35Q86; Secondary 35A01, $35 \mathrm{~B} 35$.

Keywords. Atmospheric/oceanic fluid dynamics, Semi-geostrophic equations, Quasi-geostrophic equations, Linear stability.

\section{Introduction}

The aim of this work is to derive and analyse the linearised semi-geostrophic equation (LSG in what follows) associated to a class of steady solutions of the semi-geostrophic equations (referred to as SG) on the whole space $\mathbb{R}^{3}$. We present a well-posedness theory for this equation, and discuss the stability of these steady states. For a given conservative steady solution $\nabla \bar{P}$ of SG corresponding to a time-independent strictly-convex quadratic geopotential $\bar{P}$, the associated LSG in the conservative perturbation $\nabla \psi$ is the passive transport equation

$$
\frac{\partial}{\partial t} \nabla \psi+(\bar{u} \cdot \nabla) \nabla \psi+S^{T} \nabla \psi=\mathscr{F}^{-1} \circ \mathscr{M}_{m} \circ \mathscr{F}[\nabla \psi],
$$

where the vector field $\bar{u}$ is a $\bar{P}$-dependent Eulerian velocity, $S$ is a $\bar{P}$-dependent matrix, $\mathscr{F}^{-1} \circ \mathscr{M}_{m} \circ \mathscr{F}$ is a pseudodifferential operator of order 0 with $\bar{P}$-dependent symbol $m$, and $\mathscr{F}$ is the Fourier transform, defined in (1.18). Although the existence theory in $L^{2}\left(\mathbb{R}^{3}, \mathbb{R}^{3}\right)$ of LSG follows from the theory of strongly continuous semigroups on Banach spaces, we provide a representation formula for the solution, which allows us to work with the solutions explicitly. We present and extend this result to the space of tempered distributions $S^{\prime}\left(\mathbb{R}^{3}, \mathbb{R}^{3}\right)$, to allow for the treatment of global plane-wave solutions, which are not integrable functions on the whole space $\mathbb{R}^{3}$. We also introduce concepts of stability for this family of steady solutions of the semi-geostrophic equations, by studying the long-term behaviour of wave-like solutions, in analogy with the results of [5] on the Navier-Stokes equations. Finally, we briefly mention how the same methods can be applied to the quasi-geostrophic equations, and discuss similarities and differences between the two theories.

\subsection{Contributions of this Paper}

The main novelty of this paper is the introduction of an analytical framework in which one can analyse the linearised semi-geostrophic equations for steady solutions that are globally defined in space. We consider conservative steady solutions corresponding to quadratic strictly-convex potentials: this choice simplifies the derivation, as we will see in Sect. 2, and it allows us to write the linearised equation in a very explicit manner, namely (1.1). Our choice 
of steady solutions of SG allows us to prove existence of solutions to LSG, and study the stability of the steady solutions, in a sense that we specify in Definition 1.3. It is important to notice that the derivation of LSG is only formal here: a rigorous derivation would require a well-posedness theory for the semi-geostrophic equations on the whole space $\mathbb{R}^{3}$, which is still an open problem, to the best of the authors' knowledge.

The authors believe that the framework presented in this paper can be a first step towards the understanding of the long-time behaviour of more general steady solutions of the semi-geostrophic equations, and offer an interesting first comparison between the behaviour of common steady solutions of the semi-geostrophic and the quasi-geostrophic model. In fact, in Sect. 5, we discuss how the stability analysis predicts different behaviour for some of the common steady solutions of the semi-geostrophic and the quasi-geostrophic equations.

To the best of the authors' knowledge, this is the first analysis of the stability of globally-defined steady solutions of the semi-geostrophic equations, although in the physics literature [13] the author analyses the linear stability of parallel basic flow in the baroclinic semi-geostrophic equations in geometric coordinates.

\subsection{The Semi-Geostrophic Equations in Eulerian Coordinates}

The semi-geostrophic equations can be written in various coordinate systems, depending on the analytical tools that one wishes to employ (e.g. optimal transport theory). The first formulation of SG in Eulerian coordinates was introduced in the 1950s in [9] and was studied by Hoskins in the 1970s (see [10]). However, the model attracted the interest of the mathematical community only in the late 1990s when Benamou and Brenier introduced a new formulation in [4], in a set of coordinates which are now known as geostrophic or dual coordinates, and showed that tools from the theory of optimal transport could be applied to study the well-posedness of the problem. Since then, the problem is typically studied in geostrophic coordinates, because of its connection to optimal transport theory: see, among the others, [1] and [2]. We invite the reader to see [11] for a review of the main formulations and results in the semi-geostrophic theory.

For our purposes, we shall consider SG in Eulerian coordinates, which is the original setting in which the equations were derived and studied by Hoskins [10] in 1975. In Eulerian coordinates, SG takes the form of an active transport equation given by

$$
\left\{\begin{array}{l}
\frac{\partial}{\partial t} \nabla P+(\mathcal{U}[\nabla P] \cdot \nabla) \nabla P=J\left(\nabla P-\mathrm{id}_{\Omega}\right), \\
\nabla P(\cdot, 0)=\nabla P_{0},
\end{array}\right.
$$

in the unknown time-dependent conservative vector field $\nabla P: \Omega \times \mathbb{R} \rightarrow \mathbb{R}^{3}$, where $\Omega \subseteq \mathbb{R}^{3}$. In the above, the matrix $J \in \mathbb{R}^{3 \times 3}$ is given by

$$
J:=\left(\begin{array}{lll}
0 & -1 & 0 \\
1 & 0 & 0 \\
0 & 0 & 0
\end{array}\right),
$$

and the function $\operatorname{id}_{\Omega}: x \mapsto x$ denotes the identity function on $\Omega$. The operator $\mathscr{U}: \nabla P \mapsto u$ is the formal solution operator associated to the boundary-value problem for the div-curl system on $\Omega$ given by

$$
\left\{\begin{array}{l}
\nabla \times\left(D^{2} P u\right)=\nabla \times J\left(\nabla P-\mathrm{id}_{\Omega}\right), \\
\nabla \cdot u=0 .
\end{array}\right.
$$

This boundary value problem is typically endowed with the usual no-slip condition $u \cdot n=0$ on $\partial \Omega$ if $\Omega$ is a bounded domain in $\mathbb{R}^{3}$. To guarantee well-posedness of $\mathcal{U}$ in the case when $\Omega=\mathbb{R}^{3}$, further conditions must be imposed on $u$. In this article, we are interested in the linear dynamics of SG around a special family of steady solutions in the whole space case $\Omega=\mathbb{R}^{3}$. This family of steady solutions is realised by the gradients of globally-supported quadratic strictly-convex functions: for a given symmetric positive definite matrix $A \in \operatorname{Sym}_{+}(3, \mathbb{R}) \subset \mathbb{R}^{3 \times 3}$, we consider those steady solutions $\nabla \bar{P}$ of (1.2) with

$$
\bar{P}(x):=\frac{1}{2} x \cdot A x, \quad \forall x \in \mathbb{R}^{3} .
$$


Indeed, one can show that $\nabla \bar{P}$ is a steady solution of (1.2) with corresponding velocity field $\bar{u}:=\mathscr{U}[\nabla \bar{P}]$ given by

$$
\bar{u}(x)=S x,
$$

where the matrix $S$ is given by $S:=A^{-1} J(A-I)$ and $I \in \mathbb{R}^{3 \times 3}$ is the identity matrix.

\subsection{Cullen's Stability Principle}

The choice of steady solutions (1.5) allows us to derive the linearised SG by formally taking Fréchet derivatives of (1.2) and (1.4) at $\nabla \bar{P}$ in the direction of a smooth conservative vector field $\nabla \psi$ (see Sect. 2). Using the fact that the strictly-convex $\bar{P}$ has constant Hessian, we will show that LSG can be written as the abstract Cauchy problem

$$
\left\{\begin{array}{l}
\frac{d}{d t} \phi=\mathscr{L} \phi, \\
\phi(0)=\phi_{0},
\end{array}\right.
$$

where the linear operator $\mathscr{L}$ is defined as

$$
\mathscr{L} \phi:=-(\bar{u} \cdot \nabla) \phi-S^{T} \phi+\mathscr{F}^{-1} \circ \mathscr{M}_{m} \circ \mathscr{F}[\phi],
$$

as in (1.1). In the above, the operator $\mathscr{M}_{m}$ is the multiplication operator $\mathscr{M}_{m}[\phi]=m \phi$ with symbol $m: \mathbb{R}^{3} \backslash\{0\} \rightarrow$ $\mathbb{R}$ which depends on the choice of the matrix $A$ and is given by

$$
m(x):=2 \frac{x \cdot A^{-1} J x}{x \cdot A^{-1} x}
$$

for any $x \in \mathbb{R}^{3} \backslash\{0\}$. Observe that $m \in L^{\infty}\left(\mathbb{R}^{3}\right) \cap C^{\infty}\left(\mathbb{R}^{3} \backslash\{0\}\right)$.

The primary reason we work with this specific family of steady solutions is due to the well-known Stability Principle of Cullen, which is adopted by most authors when analysing SG. Indeed, Cullen's stability principle (introduced for the first time in [7]) postulates that physically stable solutions of SG are those for which $P(\cdot, t)$ is a convex function in its spatial variable for any $t$. For more about the stability principle, we invite the reader to see [8, Ch. 3] and [6].

As an interesting consequence of our analysis in this paper, we show that whilst steady solutions of the shape (1.5) are stable in the sense of Cullen, they are in fact dynamically unstable in natural topologies. An additional structural benefit to considering steady states of the form (1.5), we show in Sect. 2, is that they result in a linearised equation that involves a pseudo-differential operator of order 0 on $\mathbb{R}^{3}$. We do not investigate the linearisation of SG around other steady solutions in this paper, and we leave it for future work. Let us also mention that the corresponding problem in bounded domains $\Omega \subset \mathbb{R}^{3}$ is substantially harder, with the existence of non-trivial steady solutions of SG (1.2) on bounded domains being an open problem.

\subsection{Characterisation of Steady Quadratic Flows}

We observed that the velocity field $\bar{u}$ induced by a geopotential $\bar{P}$ of the form (1.5) is given by the linear transformation in (1.6). In particular, different choices for the positive-definite symmetric matrix $A$ will produce different steady Eulerian flow fields. To show this, we consider a generic symmetric matrix

$$
A:=\left(\begin{array}{lll}
a & b & c \\
b & d & e \\
c & e & f
\end{array}\right),
$$

with the coefficients $a, b, c, d, e, f \in \mathbb{R}$ chosen in such a way that $A$ is positive definite. Then, the steady flow velocity is given by $\bar{u}(x)=S x$, with $S=A^{-1} J(A-I)$. In particular, $S$ can be written in terms of the coefficients of $A$ and it is easily verified that its spectrum is $\sigma(S)=\{0, \lambda,-\lambda\}$, where

$$
\lambda:=\sqrt{\frac{\mu}{\operatorname{det} A}}, \quad \mu:=a f-c^{2}+d f-e^{2}-f-\operatorname{det} A .
$$


Hence, the matrix $S$ has real eigenvalues if and only if $\mu \geq 0$, and it has two purely imaginary eigenvalues otherwise. We define the two sets in the space of positive definite symmetric real matrices

$$
\begin{aligned}
& \mathscr{A}_{+}:=\left\{A \in \operatorname{Sym}_{+}(3, \mathbb{R}): \mu>0\right\}, \\
& \mathscr{A}_{-}:=\left\{A \in \operatorname{Sym}_{+}(3, \mathbb{R}): \mu<0\right\} .
\end{aligned}
$$

As a result, the set $\mathscr{A}_{+}$corresponds to hyperbolic flows, whereas the set $\mathscr{A}_{-}$corresponds to elliptic flows. Although it is readily seen that the set $\mathscr{A}_{0}:=\left\{A \in \operatorname{Sym}_{+}(3, \mathbb{R}): \mu=0\right\}$ is non-empty, as it contains the identity matrix $I$, we do not investigate the stability of steady solutions corresponding to such matrices.

\subsection{Statement of Main Results}

For reasons pertaining to the study of plane wave perturbations that we outline in the sequel, we aim to construct solutions of (1.1) in $S^{\prime}\left(\mathbb{R}^{3}, \mathbb{R}^{3}\right)$ by duality, thereby considering the adjoint LSG given by the abstract Cauchy problem

$$
\left\{\begin{array}{l}
\frac{d}{d t} \psi=\mathscr{K} \psi, \\
\psi(0)=\psi_{0},
\end{array}\right.
$$

where the operator $\mathscr{K}$ is the adjoint of the operator $\mathscr{L}$ defined in (1.8), namely

$$
\mathscr{K} \psi:=(\bar{u} \cdot \nabla) \psi-S \psi+\mathscr{F} \circ \mathscr{M}_{m} \circ \mathscr{F}^{-1}[\psi] .
$$

The reader will observe that symbol $m$ defined in (1.9) is an even function, therefore a change of variables shows that $\mathscr{F} \circ \mathscr{M}_{m} \circ \mathscr{F}^{-1}=\mathscr{F}^{-1} \circ \mathscr{M}_{m} \circ \mathscr{F}$. This means that the analysis of the operator $\mathscr{K}$ in (1.14) is essentially the same as that of the operator $\mathscr{L}$ defined in (1.8), up to a change of sign in the first term and transposing the matrix $S$ in the second. In particular, all of the results that we prove for $\mathscr{L}$ hold for $\mathscr{K}$ mutatis mutandis.

Remark 1.1. Since the velocity $\bar{u}$ corresponding to the steady solution (1.5) is given by $\bar{u}(x)=S x$, the associated flow map $F_{\bar{u}}(x, t)=e^{t S} x$ is a volume-preserving diffeomorphism of $\mathbb{R}^{3}$ for any time $t$, and we expect LSG in Eulerian coordinates (1.1) to be equivalent to the formulation in Lagrangian coordinates. We will not comment further on the Lagrangian formulation, except for mentioning that the existence of strong solutions of LSG in Lagrangian coordinates can be proved in $L^{2}\left(\mathbb{R}^{3}, \mathbb{R}^{3}\right)$ by applying the theory of evolution systems generated by time-dependent operators, as presented in [12, Ch. 5]. In fact, LSG in Lagrangian coordinates (up to a rescaling) is given by the abstract Cauchy problem

$$
\left\{\begin{array}{l}
\frac{d}{d t} \Phi(t)=\mathcal{A}(t) \Phi(t), \\
\Phi(0)=\Phi_{0},
\end{array}\right.
$$

where the time-depending operator $\mathcal{A}(t)$ is defined as

$$
\mathcal{A}(t):=\mathscr{F}^{-1} \circ \mathscr{M}_{\widetilde{m}(t)} \circ \mathscr{F},
$$

with the symbol $\tilde{m}$ defined in (1.19) in the next section.

As we mentioned above, our purpose is firstly to present the existence theory of LSG in $L^{2}\left(\mathbb{R}^{3}, \mathbb{R}^{3}\right)$ and extend it to tempered distributions in $S^{\prime}\left(\mathbb{R}^{3}, \mathbb{R}^{3}\right)$. The following is our first main result:

Theorem 1.2. Given an initial tempered distribution $\eta_{0} \in S^{\prime}\left(\mathbb{R}^{3}, \mathbb{R}^{3}\right)$, there exists a strong solution in $S^{\prime}\left(\mathbb{R}^{3}, \mathbb{R}^{3}\right)$ of (1.7), in the sense of Definition 1.7 (iv).

The reason we extend the existence theory of LSG to allow for less regular solutions than those which take values in $L^{2}\left(\mathbb{R}^{3}, \mathbb{R}^{3}\right)$ is so that our solution theory admits plane-wave solutions of the form

$$
\phi(x, t)=\nabla f(x, t), \quad f(x, t)=\frac{a(t)}{2 \pi} \sin (2 \pi k(t) \cdot x),
$$

where $a: \mathbb{R} \rightarrow \mathbb{R}$ and $k: \mathbb{R} \rightarrow \mathbb{R}^{3}$ are appropriately chosen functions. These solutions are not integrable on $\mathbb{R}^{3}$, with $\phi(\cdot, t) \notin L^{p}\left(\mathbb{R}^{3}, \mathbb{R}^{3}\right)$ for any $p \in[1, \infty)$. Nevertheless, we consider the corresponding time-dependent regular 
distribution $\eta(t)=\eta_{\phi(\cdot, t)}$ and characterise the functions $a$ and $k$ for which $\eta$ is a strong solution of LSG in $S^{\prime}$, as in the sense that we clarify in Definition 1.7 (v). Then, for these specific solutions, we look at their stability in the following sense.

Definition 1.3. We say that a steady solution $\nabla \bar{P}$ of SG (1.2) is stable to plane-wave perturbations if any planewave solution $\phi$ of the associated LSG (1.1) belongs to $L^{\infty}\left(\mathbb{R}^{3} \times \mathbb{R}, \mathbb{R}^{3}\right)$. Otherwise, we say that $\nabla \bar{P}$ is unstable to plane-wave perturbations, i.e. there exists a plane-wave solution $\phi$ of LSG such that $\|\phi(\cdot, t)\|_{L^{\infty}\left(\mathbb{R}^{3}, \mathbb{R}^{3}\right)}$ is unbounded.

With this in place, let us state the main stability result of this work.

Theorem 1.4. The steady solution $\nabla \bar{P}$ defined in (1.5) associated to a matrix $A$ is stable to plane-wave perturbations if it corresponds to an elliptic flow, i.e. $A \in \mathscr{A}_{-}$as in (1.12), and it is unstable to plane-wave perturbations if it corresponds to a hyperbolic flow, i.e. $A \in \mathscr{A}_{+}$.

\subsection{Notation and Main Definitions}

For the reader's convenience, we fix here the notation that will be used throughout. The space $S\left(\mathbb{R}^{3}, \mathbb{R}^{3}\right)$ is the space of Schwartz functions, i.e. the set of all $f \in C^{\infty}\left(\mathbb{R}^{3}, \mathbb{R}^{3}\right)$ such that

$$
\|f\|_{\alpha, \beta}:=\sup _{x \in \mathbb{R}^{3}}\left|x^{\alpha} D^{\beta} f(x)\right|<\infty,
$$

for any $\alpha, \beta \in \mathbb{N}^{3}$ multi-indices, where $x^{\alpha}:=x_{1}^{\alpha_{1}} x_{2}^{\alpha_{2}} x_{3}^{\alpha_{3}}$ and $D^{\beta}=\frac{\partial^{|\beta|}}{\partial x_{1}^{\beta_{1}} \partial x_{2}^{\beta_{2}} \partial x_{3}^{\beta_{3}}}$. The set $S\left(\mathbb{R}^{3}, \mathbb{R}^{3}\right)$ endowed with the weak topology generated by the family of seminorms $\left\{\|\cdot\|_{\alpha, \beta}\right\}_{\alpha, \beta \in \mathbb{N}^{3}}$ is a Fréchet space. One can prove that the topology generated by the family of seminorms is equivalent to the topology generated by the family of their finite linear combinations. The space $S^{\prime}\left(\mathbb{R}^{3}, \mathbb{R}^{3}\right)$ is the space of tempered distributions endowed with the weak ${ }^{*}$ topology. We use the notation $S$ and $S^{\prime}$ in place of $S\left(\mathbb{R}^{3}, \mathbb{R}^{3}\right)$ and $S^{\prime}\left(\mathbb{R}^{3}, \mathbb{R}^{3}\right)$ respectively, unless it is necessary to specify a different range of the Schwartz functions. $\mathscr{F}$ and $\mathscr{F}^{-1}$ denote the Fourier transform and its inverse, respectively. To avoid confusion due to the notation, we specify that we define the Fourier transform and its inverse on $L^{1}\left(\mathbb{R}^{3}, \mathbb{R}^{3}\right)$ as follows:

$$
\mathscr{F}[\phi](\xi):=\int_{\mathbb{R}^{3}} \phi(x) e^{-2 \pi i \xi \cdot x} d x, \quad \mathscr{F}^{-1}[\phi](x):=\int_{\mathbb{R}^{3}} \phi(\xi) e^{2 \pi i \xi \cdot x} d \xi,
$$

and extend this definition to $L^{2}\left(\mathbb{R}^{3}, \mathbb{R}^{3}\right)$ by density in the usual way. We also use the notation $\hat{\phi}:=\mathscr{F}[\phi]$ and $\check{\phi}:=\mathscr{F}^{-1}[\phi]$.

In order to construct solutions to LSG, we will need to consider the following Fourier symbols, defined in terms of the symbol $m$ in (1.9):

$$
\begin{array}{ll}
\widetilde{m}(t ; \xi):=m\left(e^{\left.-t S^{T} \xi\right),} \quad \widetilde{M}(t ; \xi):=e^{\int_{0}^{t} \widetilde{m}(r ; \xi) d r},\right. \\
\bar{m}(t ; \xi):=m\left(e^{\left.t S^{T} \xi\right),} \quad \bar{M}(t ; \xi):=e^{\int_{0}^{t} \bar{m}(r ; \xi) d r} .\right.
\end{array}
$$

For any $t \in \mathbb{R}, \widetilde{m}(t ; \cdot)$ is an even function on $\mathbb{R}^{3}$, therefore $\mathscr{F}^{-1} \circ \mathscr{M}_{\widetilde{m}(t ; \cdot)} \circ \mathscr{F}=\mathscr{F} \circ \mathscr{M}_{\widetilde{m}(t ; \cdot)} \circ \mathscr{F}^{-1}$, and the same holds for $\bar{m}(t ; \cdot), \widetilde{M}(t ; \cdot)$ and $\bar{M}(t ; \cdot)$.

The operator $\mathscr{L}$ defined in (1.8) consists of three additive terms: we will need to refer to them and the groups of operators that they generate, therefore we list here their definitions for the reader's convenience. We formally define the following operators without specifying their domains, as we discuss in Remark 1.6.

Definition 1.5. (i) We consider the three additive terms that constitute the operator $\mathscr{L}=L_{1}+L_{2}+L_{3}$ defined in (1.8):

$$
\begin{aligned}
& L_{1}[\phi]:=-(\bar{u} \cdot \nabla) \phi, \\
& L_{2}[\phi]:=-S^{T} \phi,
\end{aligned}
$$




$$
L_{3}[\phi]:=\mathscr{F}^{-1} \circ \mathscr{M}_{m} \circ \mathscr{F}[\phi] .
$$

(ii) We define the families of operators $\left\{T_{1}(t)\right\}_{t \in \mathbb{R}},\left\{T_{2}(t)\right\}_{t \in \mathbb{R}}$ and $\left\{T_{3}(t)\right\}_{t \in \mathbb{R}}$ as follows:

$$
\begin{aligned}
& T_{1}(t)[\phi](x)=\phi\left(e^{-t S} x\right), \\
& T_{2}(t)[\phi](x)=e^{-t S^{T}} \phi(x), \\
& T_{3}(t)[\phi](x)=\mathscr{F}^{-1} \circ \mathscr{M}_{\widetilde{M}(t)} \circ \mathscr{F}[\phi](x) .
\end{aligned}
$$

(iii) For any $t \in \mathbb{R}$, we define the operator $G: t \mapsto G(t)[\phi]:=T_{1}(t) T_{2}(t) T_{3}(t)[\phi]$, i.e.

$$
G(t)[\phi](x)=\int_{\mathbb{R}^{3}} e^{-t S^{T}} \hat{\phi}(\xi) \widetilde{M}(t ; \xi) e^{2 \pi i \xi \cdot e^{-t S} x} d \xi .
$$

Remark 1.6. With an abuse of notation, we use the same symbol to denote an operator defined in different topologies, as the topology with which we work will be clear by the context. For instance, we denote by $\mathscr{L}$ both the operator in (1.8) defined on the space of distributions $S^{\prime}$ and its restriction to functions in $L^{2}\left(\mathbb{R}^{3}, \mathbb{R}^{3}\right)$ and $S$. By this, we mean that if $\eta_{f}$ is a regular distribution corresponding to the function $f \in L^{2}\left(\mathbb{R}^{3}, \mathbb{R}^{3}\right)$, then we write $\mathscr{L} \eta_{f}=\eta_{\mathscr{L} f}$. For the sake of clarity, we mention that by regular distribution we mean a distribution $\eta \in \mathcal{S}^{\prime}$ such that there exists a function $f \in L^{p}\left(\mathbb{R}^{3}, \mathbb{R}^{3}\right)$ for some $p \in[1, \infty]$, for which

$$
\langle\eta, \psi\rangle=\int_{\mathbb{R}^{3}} f(x) \cdot \psi(x) d x, \quad \forall \psi \in S .
$$

Some concepts of the theory of semigroups will be employed throughout the paper: for instance, the reader can observe that $L_{1}$ is the infinitesimal generator of the strongly continuous group $\left\{T_{1}(t)\right\}_{t \in \mathbb{R}}$ in $L^{2}\left(\mathbb{R}^{3}, \mathbb{R}^{3}\right)$. The theory of semigroups on Banach spaces is classical (and can be found in [12]), whilst the theory of semigroups on locally convex topological spaces can be found in [15, Ch. IX] and in [3].

We now introduce the notions of solutions that will be of our interest in the following sections.

Definition 1.7. (i) We say that a function $\phi: \mathbb{R} \rightarrow L^{2}\left(\mathbb{R}^{3}, \mathbb{R}^{3}\right)$ is a strong solution in $L^{2}\left(\mathbb{R}^{3}, \mathbb{R}^{3}\right)$ of LSG (1.7) if $\phi \in C^{1}\left(\mathbb{R}, L^{2}\left(\mathbb{R}^{3}, \mathbb{R}^{3}\right)\right)$ and it satisfies (1.7) in $L^{2}\left(\mathbb{R}^{3}, \mathbb{R}^{3}\right)$ for all $t \in \mathbb{R}$.

(ii) We say that a function $\phi$ is a weak solution in $L^{2}\left(\mathbb{R}^{3}, \mathbb{R}^{3}\right)$ of $\operatorname{LSG}(1.7)$ if $\phi \in C^{0}\left(\mathbb{R}, L^{2}\left(\mathbb{R}^{3}, \mathbb{R}^{3}\right)\right.$ ), it satisfies the following integral equation

$$
\begin{aligned}
& \int_{\mathbb{R}} \int_{\mathbb{R}^{3}} \phi(x, t) \cdot\left[\frac{\partial}{\partial t} f(x, t)+(\bar{u}(x) \cdot \nabla) f(x, t)-S f(x, t)\right. \\
& \left.\quad+\mathscr{F}^{-1} \circ \mathscr{M}_{m} \circ \mathscr{F}[f(\cdot, t)](x)\right] d x d t=0,
\end{aligned}
$$

for any test function $f \in C_{C}^{\infty}\left(\mathbb{R}^{3} \times \mathbb{R}, \mathbb{R}^{3}\right)$, and $\phi(\cdot, 0)=\phi_{0}$ in $L^{2}\left(\mathbb{R}^{3}, \mathbb{R}^{3}\right)$.

(iii) We say that a function $\phi: \mathbb{R} \rightarrow S$ is a strong solution in $S$ of LSG (1.7) if $\phi \in C^{1}(\mathbb{R}, S)$ and it satisfies the differential equation and the initial condition in (1.7) in $S$ for all $t \in \mathbb{R}$.

(iv) We say that $\eta: \mathbb{R} \rightarrow S^{\prime}$ is a strong solution in $S^{\prime}$ of LSG (1.7) if $\eta \in C^{1}\left(\mathbb{R}, S^{\prime}\right)$ and it satisfies the differential equation and the initial condition in (1.1) in $S^{\prime}$ for all $t \in \mathbb{R}$, i.e.

$$
\left\langle\frac{d}{d t} \eta(t), \psi\right\rangle=\langle\mathscr{L} \eta(t), \psi\rangle \quad \text { and } \quad\langle\eta(0), \psi\rangle=\left\langle\eta_{0}, \psi\right\rangle,
$$

for any $\psi \in S$ and $t \in \mathbb{R}$. The time derivative in the expression above is to be interpreted in the weak ${ }^{*}$-sense in $S^{\prime}$.

(v) A function $\phi: \mathbb{R}^{3} \times \mathbb{R} \rightarrow \mathbb{R}$ is a plane-wave solution of LSG (1.7) if there exist two functions $a \in C^{1}(\mathbb{R}, \mathbb{R})$ and $k \in C^{1}\left(\mathbb{R}, \mathbb{R}^{3}\right)$ such that $\phi$ is of the form (1.16) and the time-dependent regular distribution $\eta_{\phi(\cdot, t)}$ is a strong solution in $S^{\prime}$ of LSG corresponding to the regular initial datum $\eta_{\phi(\cdot, 0)}$, according to the definition (iv) above. 


\subsection{Structure of the Paper}

We present the proofs of the theorems above in the following sections, proceeding as follows: in Sect. 2, we present the formal derivation of LSG for a conservative vector field, starting from SG as written in (1.2), and we comment on the degeneracy of the symbol $m$, distinguishing between the matrices $A$ that give rise to a trivial pseudo-differential operator and those that do not. In Sect. 3, we start by presenting the existence theory for solutions in $L^{2}\left(\mathbb{R}^{3}, \mathbb{R}^{3}\right)$, and then provide the existence result in the space of Schwartz functions $S$. We conclude the section with the Proof of Theorem 1.2 and discuss the regularity of solutions with regular initial datum, connecting the statement with the existence in $L^{2}\left(\mathbb{R}^{3}, \mathbb{R}^{3}\right)$. In Sect. 4 , we focus on plane-wave solutions of LSG and look at their long-term behaviour in the case of both elliptic and hyperbolic flows, proving Theorem 1.4. In Sect. 5, we briefly investigate the stability of the same family of steady solutions in the quasi-geostrophic theory, and draw a comparison of the results in the two cases.

\section{Derivation of LSG}

We provide a formal derivation of LSG in Eulerian coordinates, as in (1.1), from SG in the form presented in (1.2). We observe that SG can be written as the abstract Cauchy problem

$$
\left\{\begin{array}{l}
\frac{d}{d t} \nabla P=\mathcal{N}[\nabla P], \\
\nabla P(0)=\nabla P_{0},
\end{array}\right.
$$

where the nonlinear operator $\mathcal{N}$ is defined formally as

$$
\mathscr{N}[\nabla P]:=-\left(D^{2} P\right)^{T} \mathscr{U}[\nabla P]+J\left(\nabla P-\mathrm{id}_{\mathbb{R}^{3}}\right) .
$$

Therefore, the linearised SG at $\nabla \bar{P}$ is obtained by formally taking the Fréchet derivative of the operator $\mathcal{N}$ at $\nabla \bar{P}$ and it corresponds to the linear abstract Cauchy problem of the form

$$
\left\{\begin{array}{l}
\frac{d}{d t} \nabla \psi=d \mathscr{N}[\nabla \bar{P} ; \nabla \psi], \\
\nabla \psi(0)=\nabla \psi_{0},
\end{array}\right.
$$

in the unknown conservative perturbation $\nabla \psi \in C^{\infty}(\mathbb{R}, S)$.

Proposition 2.1. Given the smooth steady solution $\nabla \bar{P}$ of $S G$ defined in (1.5), the formal Fréchet derivative of the operator $\mathcal{N}$ defined in (2.1) at $\nabla \bar{P}$ acting on a smooth time-dependent conservative vector field $\nabla \psi$ can be written as the operator $\mathscr{L}$ defined in (1.8).

Proof. Formally, the Fréchet derivative of the operator $\mathcal{N}$ at $\nabla \bar{P}$ in the direction $\nabla \psi$ is given by

$$
d \mathscr{N}[\nabla \bar{P} ; \nabla \psi]=-A d \mathscr{U}[\nabla \bar{P} ; \nabla \psi]-D^{2} \psi \bar{u}+J \nabla \psi,
$$

where the linear operator $d \mathscr{U}[\nabla \bar{P} ; \cdot]$ is the Fréchet derivative of the nonlinear operator $\mathcal{U}$, associated to the div-curl system (1.4), at the steady solution $\nabla \bar{P}$ in the direction $\nabla \psi$. The vector field $\bar{u}$ is the steady flow velocity defined in (1.6). One can show that $v:=d \mathscr{U}[\nabla \bar{P} ; \nabla \psi]$ coincides with a solution of the div-curl system

$$
\left\{\begin{array}{l}
\nabla \times(A v)=-\nabla \times\left(D^{2} \psi \bar{u}\right)+\nabla \times J \nabla \psi \\
\nabla \cdot v=0
\end{array}\right.
$$

We consider $d \mathscr{N}[\nabla \bar{P} ; \nabla \psi]$, defined in (2.3), and observe in (2.4) that it is a curl-free quantity, therefore there exists a scalar function $E$ such that

$$
\nabla E=-A d \mathscr{U}[\nabla \bar{P} ; \nabla \psi]-D^{2} \psi \bar{u}+J \nabla \psi .
$$

As the matrix $A$ is positive definite, and $d \mathscr{U}[\nabla \bar{P} ; \nabla \psi]$ is divergence-free, we can write an elliptic equation for $E$ in divergence form

$$
\nabla \cdot\left(A^{-1} \nabla E\right)=F_{1}+F_{2}:=-\nabla \cdot\left(A^{-1} D^{2} \psi \bar{u}\right)+\nabla \cdot\left(A^{-1} J \nabla \psi\right)
$$


By formally taking Fourier transform across the above equality, we have that

$$
\widehat{E}=\widehat{E}_{1}+\widehat{E}_{2}, \quad \widehat{E}_{i}(\xi):=-\frac{1}{4 \pi^{2} \xi \cdot A^{-1} \xi} \widehat{F}_{i}(\xi) .
$$

We start looking at $E_{1}$ : by considering the Fourier transform of $F_{1}$, namely

$$
\begin{aligned}
\widehat{F_{1}}(\xi) & =-\int_{\mathbb{R}^{3}} \nabla \cdot\left(A^{-1} D^{2} \psi(x) S x\right) e^{-2 \pi i \xi \cdot x} d x \\
& =2 \pi i S A^{-1} \xi \cdot \widehat{\nabla \psi}(\xi)+4 \pi^{2}\left(\xi \cdot A^{-1} \xi\right) \mathscr{F}[\bar{u} \cdot \nabla \psi](\xi),
\end{aligned}
$$

one can write the explicit formula for $E_{1}$ :

$$
\begin{aligned}
E_{1}(x) & =-\frac{1}{4 \pi^{2}} \int_{\mathbb{R}^{3}} \frac{\widehat{F_{1}}(\xi)}{\xi \cdot A^{-1} \xi} e^{2 \pi i \xi \cdot x} d \xi \\
& =\frac{1}{2 \pi i} \int_{\mathbb{R}^{3}} \frac{S A^{-1} \xi \cdot \widehat{\nabla \psi}(\xi)}{\xi \cdot A^{-1} \xi} e^{2 \pi i \xi \cdot x} d \xi-S x \cdot \nabla \psi(x) \\
& =\int_{\mathbb{R}^{3}} \frac{\xi \cdot S A^{-1} \xi}{\xi \cdot A^{-1} \xi} \widehat{\psi}(\xi) e^{2 \pi i \xi \cdot x} d \xi-S x \cdot \nabla \psi(x) .
\end{aligned}
$$

Hence, one can calculate

$$
\nabla E_{1}(x)=\int_{\mathbb{R}^{3}} \frac{\xi \cdot S A^{-1} \xi}{\xi \cdot A^{-1} \xi} \widehat{\nabla \psi}(\xi) e^{2 \pi i \xi \cdot x} d \xi-S^{T} \nabla \psi(x)-(S x \cdot \nabla) \nabla \psi(x) .
$$

Similarly, to find $E_{2}$, we first write

$$
\begin{aligned}
\widehat{F_{2}}(\xi) & =\int_{\mathbb{R}^{3}} \nabla \cdot\left(A^{-1} J \nabla \psi(x)\right) e^{-2 \pi i \xi \cdot x} d x \\
& =-2 \pi i J A^{-1} \xi \cdot \widehat{\nabla \psi}(\xi),
\end{aligned}
$$

and compute the inverse Fourier transform to find the explicit formula for $E_{2}$ given by

$$
\begin{aligned}
E_{2}(x) & =-\frac{1}{2 \pi i} \int_{\mathbb{R}^{3}} \frac{J A^{-1} \xi \cdot \widehat{\nabla \psi}(\xi)}{\xi \cdot A^{-1} \xi} e^{2 \pi i \xi \cdot x} d \xi \\
& =-\int_{\mathbb{R}^{3}} \frac{\xi \cdot J A^{-1} \xi}{\xi \cdot A^{-1} \xi} \widehat{\psi}(\xi) e^{2 \pi i \xi \cdot x} d \xi .
\end{aligned}
$$

Therefore, we find

$$
\nabla E_{2}(x)=-\int_{\mathbb{R}^{3}} \frac{\xi \cdot J A^{-1} \xi}{\xi \cdot A^{-1} \xi} \widehat{\nabla \psi}(\xi) e^{2 \pi i \xi \cdot x} d \xi .
$$

We have now a representation formula for $\nabla E$ by the expression above and (2.6):

$$
\nabla E(x)=-(S x \cdot \nabla) \nabla \psi(x)-S^{T} \nabla \psi(x)+\int_{\mathbb{R}^{3}} \frac{\xi \cdot(S-J) A^{-1} \xi}{\xi \cdot A^{-1} \xi} \widehat{\nabla \psi}(\xi) e^{2 \pi i \xi \cdot x} d \xi .
$$

To obtain (1.1), we just need to observe that

$$
\xi \cdot(S-J) A^{-1} \xi=2\left(\xi \cdot A^{-1} J \xi\right) .
$$

Remark 2.2. (Degeneracy of the pseudo-differential operator) The flow of LSG is interesting due to the presence of the pseudo-differential operator $\mathscr{F}^{-1} \circ \mathscr{M}_{m} \circ \mathscr{F}$, so we show that the action of this operator is not trivial, i.e. we show that the pseudo-differential operator does not vanish for all choices of the matrix $A$. In fact, we have that $\mathscr{F}^{-1} \circ \mathscr{M}_{m} \circ \mathscr{F}[\nabla \psi]=0$ for a non-trivial $\nabla \psi$ if and only if $m=0$ a.e. on $\mathbb{R}^{3}$. Therefore, we have that the operator $\mathscr{F}^{-1} \circ \mathscr{M}_{m} \circ \mathscr{F}$ is the zero operator if the matrix $A$ belongs to the set of matrices

$$
\mathscr{B}:=\left\{A \in \operatorname{Sym}_{+}(3, \mathbb{R}): x \cdot\left(A^{-1} J\right) x=0 \forall x \in \mathbb{R}^{3}\right\} .
$$


We denote the complement of $\mathscr{B}$ by $\mathscr{G}:=\operatorname{Sym}_{+}(3, \mathbb{R}) \backslash \mathscr{B}$. An example of a matrix in the set $\mathscr{B}$ is $A=\beta I$, that describes a non-trivial elliptic flow when $\beta \in(0,1) \cup(1, \infty)$ : indeed, the corresponding steady geopotential and steady flow velocity field are given respectively by

$$
\bar{P}_{\beta}(x)=\frac{\beta}{2}|x|^{2}, \quad \bar{u}_{\beta}(x)=\frac{\beta-1}{\beta} J x .
$$

In particular, the matrices in the set $\mathscr{B}$ can generate both elliptic and hyperbolic flows, defined in (1.12).

\section{Existence Theory for LSG}

In this section, we present the existence results for LSG in $L^{2}\left(\mathbb{R}^{3}, \mathbb{R}^{3}\right)$, then in $S$, and finally in $S^{\prime}$, where we define strong solutions by duality. In fact, as we mentioned before, the operator $\mathscr{L}$ defined in (1.8) and the operator $\mathscr{L}^{\prime}=\mathscr{K}$ defined in (1.14) are the same up to a sign and the transposition of the matrix $S$, therefore any result for LSG (1.7) can be mirrored for the abstract Cauchy problem (1.13).

Although the existence of solutions in $L^{2}\left(\mathbb{R}^{3}, \mathbb{R}^{3}\right)$ can be proved by using the theory of strongly continuous semigroups on Banach spaces, we give a direct proof using the explicit representation formula of the solution. Formally, given the initial datum $\phi_{0}$, the solution $\phi$ can be written as $\phi(\cdot, t)=G(t) \phi_{0}$, where the operator $G(t)$ is defined in (1.21). We provide here a formal derivation of the formula.

First of all, we derive LSG in Lagrangian coordinates, which we introduced in (1.15). Given a smooth solution $\phi$ of LSG (1.7), we define the function

$$
\Phi(x, t):=e^{t S^{T}} \phi\left(e^{t S} x, t\right), \quad(x, t) \in \mathbb{R}^{3} \times \mathbb{R},
$$

and observe that

$$
\begin{aligned}
\frac{\partial}{\partial t} \Phi(x, t) & =\frac{\partial}{\partial t}\left(e^{t S^{T}} \phi\left(e^{t S} x, t\right)\right) \\
& =S^{T} e^{t S^{T}} \phi\left(e^{t S} x, t\right)+e^{t S^{T}} \frac{\partial}{\partial t} \phi\left(e^{t S} x, t\right)+e^{t S^{T}}\left(\frac{\partial}{\partial t} e^{t S} x \cdot \nabla\right) \phi\left(e^{t S} x, t\right) \\
& =e^{t S^{T}}\left[\frac{\partial}{\partial t} \phi\left(e^{t S} x, t\right)+S^{T} \phi\left(e^{t S} x, t\right)+\left(S e^{t S} x \cdot \nabla\right) \phi\left(e^{t S} x, t\right)\right] \\
& =e^{t S^{T}}\left[\mathscr{F}^{-1} \circ \mathscr{M}_{m} \circ \mathscr{F}[\phi(\cdot, t)]\left(e^{t S} x\right)\right] \\
& =e^{t S^{T}} \int_{\mathbb{R}^{3}} \int_{\mathbb{R}^{3}} m(\xi) \phi(y, t) e^{-2 \pi i y \cdot \xi} e^{2 \pi i \xi \cdot e^{t S} x} d y d \xi \\
& =\int_{\mathbb{R}^{3}} \int_{\mathbb{R}^{3}} m(\xi) \Phi\left(e^{-t S} y, t\right) e^{-2 \pi i y \cdot \xi} e^{2 \pi i \xi \cdot e^{t S} x} d y d \xi \\
& =\int_{\mathbb{R}^{3}} \int_{\mathbb{R}^{3}} \tilde{m}(t ; \xi) \Phi(y, t) e^{-2 \pi i y \cdot \xi} e^{2 \pi i \xi \cdot x} d y d \xi \\
& =\mathscr{F}^{-1} \circ \mathscr{M}_{\widetilde{m}(t ; \cdot)} \circ \mathscr{F}[\Phi(\cdot, t)](x),
\end{aligned}
$$

which corresponds to the Eq. (1.15). We can now formally take the Fourier transform to write explicitly the solution $\Phi$ : we have that $\Phi$ solves LSG in Lagrangian coordinates (1.15) if and only if $\widehat{\Phi(\cdot, t)}$ solves the abstract Cauchy problem

$$
\left\{\begin{array}{l}
\frac{d}{d t} \widehat{\Phi(\cdot, t)}=\widetilde{m}(t) \widehat{\Phi(\cdot, t)}, \\
\frac{\Phi(\cdot, 0)}{=\phi_{0}},
\end{array}\right.
$$

If we multiply both the sides of the differential equation by the integrating factor $\widetilde{M}(t ; \cdot)^{-1}=e^{-\int_{0}^{t} \widetilde{m}(r \cdot \cdot) d r}$, we see that $\Phi(\cdot, t)=\mathscr{F}^{-1}\left[\widetilde{M}(t ; \cdot) \widehat{\phi_{0}}\right]$ is the solution of LSG in Lagrangian coordinates (1.15). By the definition of the function $\Phi$ in (3.1), we have that 


$$
\begin{aligned}
\phi(x, t) & =e^{-t S^{T}} \Phi\left(e^{-t S} x, t\right) \\
& =\int_{\mathbb{R}^{3}} \widetilde{M}(t ; \xi) e^{-t S^{T}} \widehat{\phi_{0}}(\xi) e^{2 \pi i \xi \cdot e^{-t S} x} d \xi \\
& =G(t) \phi_{0}(x) .
\end{aligned}
$$

The derivation above is formal, and we now prove that $G(t) \phi_{0}$ is indeed a solution of LSG in the appropriate topologies, starting with $L^{2}\left(\mathbb{R}^{3}, \mathbb{R}^{3}\right)$.

\subsection{Existence of Solutions of LSG in $L^{2}\left(\mathbb{R}^{3}, \mathbb{R}^{3}\right)$}

Theorem 3.1. Given $\phi_{0} \in L^{2}\left(\mathbb{R}^{3}, \mathbb{R}^{3}\right)$, the function $\phi(\cdot, t):=G(t) \phi_{0}$, where the operator $G(t)$ is defined in (1.21), is a weak solution in $L^{2}\left(\mathbb{R}^{3}, \mathbb{R}^{3}\right)$ of $L S G(1.7)$. Moreover, if $\phi_{0}$ belongs to the domain

$$
D(\mathscr{L}):=\left\{\phi \in L^{2}\left(\mathbb{R}^{3}, \mathbb{R}^{3}\right): \mathscr{L} \phi \in L^{2}\left(\mathbb{R}^{3}, \mathbb{R}^{3}\right)\right\}
$$

of the operator $\mathscr{L}$, then $\phi$ is the unique strong solution of $L S G$ in $L^{2}\left(\mathbb{R}^{3}, \mathbb{R}^{3}\right)$. Furthermore, if $\phi_{0}$ is conservative, namely $\phi_{0}=\nabla f_{0}$ for some $f_{0} \in H^{1}\left(\mathbb{R}^{3}\right)$, then $G(t) \phi_{0}$ is conservative for all times $t \in \mathbb{R}$.

We can observe from Definition 1.5 of the operators $L_{1}, L_{2}$ and $L_{3}$ that the domain $D(\mathscr{L})$ corresponds to

$$
D\left(L_{1}\right)=\left\{\phi \in L^{2}\left(\mathbb{R}^{3}, \mathbb{R}^{3}\right):(\bar{u} \cdot \nabla) \phi \in L^{2}\left(\mathbb{R}^{3}, \mathbb{R}^{3}\right)\right\},
$$

as the operators $L_{2}$ and $L_{3}$ are bounded on $L^{2}\left(\mathbb{R}^{3}, \mathbb{R}^{3}\right)$.

Before proving the theorem above, we need the following auxiliary result.

Lemma 3.2. For any $t \in \mathbb{R}$, the two operators $\mathscr{L}$ and $G(t)$, defined in (1.8) and (1.21), respectively, commute on the domain $D(\mathscr{L})$.

Proof. In Definition 1.5, we wrote the linear operator $\mathscr{L}$ as the sum of three operators $\mathscr{L}=L_{1}+L_{2}+L_{3}$, and we defined $G(t)=T_{1}(t) T_{2}(t) T_{3}(t)$. One can easily notice that $L_{2}$ and $T_{2}(t)$ are just given by multiplication by $S^{T}$ and $e^{t S^{T}}$ respectively, and that they commute with all the other operators involved. Therefore, it suffices to prove that the operator

$$
T_{1}(t) T_{3}(t) L_{1}+T_{1}(t) T_{3}(t) L_{3}-L_{1} T_{1}(t) T_{3}(t)-L_{3} T_{1}(t) T_{3}(t)
$$

is the zero operator on $D(\mathscr{L})$. We start by looking at the first term. For any $\phi \in D(\mathscr{L})$ and $x \in \mathbb{R}^{3}$ we have the following:

$$
\begin{aligned}
& T_{1}(t) T_{3}(t) L_{1}[\phi](x) \\
& =\int_{\mathbb{R}^{3}} \widetilde{M}(t ; \xi)\left(S^{T} \xi \cdot \nabla\right) \hat{\phi}(\xi) e^{2 \pi i \xi \cdot e^{-t S} x} d \xi \\
& \quad+\operatorname{tr}(S) T_{1}(t) T_{3}(t)[\phi](x) .
\end{aligned}
$$

The second term in (3.3) is given by the composition of two pseudo-differential operators: by standard calculations, one can show that for any $\phi \in D(\mathscr{L})$ the following holds:

$$
\begin{aligned}
& T_{1}(t) T_{3}(t) L_{3}[\phi](x) \\
& =\left(\mathscr{F}^{-1} \circ \mathscr{M}_{\widetilde{M}(t)} \circ \mathscr{F} \circ \mathscr{F}^{-1} \circ \mathscr{M}_{m} \circ \mathscr{F}[\phi]\right)\left(e^{-t S} x\right) \\
& =\int_{\mathbb{R}^{3}} \widetilde{M}(t ; \xi) m(\xi) \hat{\phi}(\xi) e^{2 \pi i \xi \cdot e^{-t S} x} d \xi .
\end{aligned}
$$

For the third term in (3.3), we consider the following: 


$$
\begin{aligned}
L_{1} T_{1}(t) T_{3}(t)[\phi](x)= & -(S x \cdot \nabla) \int_{\mathbb{R}^{3}} \widetilde{M}(t ; \xi) \hat{\phi}(\xi) e^{2 \pi i \xi \cdot e^{-t S} x} d \xi \\
= & -\int_{\mathbb{R}^{3}} \widetilde{M}(t ; \xi) \widehat{\phi}(\xi) S^{T} \xi \cdot \nabla_{\xi} e^{2 \pi i \xi \cdot e^{-t S} x} d \xi \\
= & \int_{\mathbb{R}^{3}} S^{T} \xi \cdot \nabla \widetilde{M}(t ; \xi) \widehat{\phi}(\xi) e^{2 \pi i \xi \cdot e^{-t S} x} d \xi \\
& +\int_{\mathbb{R}^{3}} \widetilde{M}(t ; \xi)\left(S^{T} \xi \cdot \nabla\right) \hat{\phi}(\xi) e^{2 \pi i \xi \cdot e^{-t S} x} d \xi \\
& +\operatorname{tr}(S) T_{1}(t) T_{3}(t)[\phi](x) .
\end{aligned}
$$

In the last term in (3.3), we have again the composition of two pseudo-differential operators: by using their definition, one can easily see that

$$
\begin{aligned}
L_{3} & T_{1}(t) T_{3}(t)[\phi](x) \\
& =\int_{\left(\mathbb{R}^{3}\right)^{3}} m(\xi) \widetilde{M}(t ; \eta) \hat{\phi}(\eta) e^{2 \pi i \eta \cdot e^{-t S} y} e^{2 \pi i y \cdot \xi} e^{-2 \pi i \xi \cdot x} d \eta d y d \xi \\
& =\int_{\mathbb{R}^{3}} \tilde{m}(t ; \eta) \widetilde{M}(t ; \eta) \hat{\phi}(\eta) e^{2 \pi i \eta \cdot e^{-t S} x} d \eta .
\end{aligned}
$$

Therefore, the operator in (3.3) is simply given by the following pseudo-differential operator:

$$
\begin{aligned}
& \left(T_{1}(t) T_{3}(t) L_{1}+T_{1}(t) T_{3}(t) L_{3}-L_{1} T_{1}(t) T_{3}(t)-L_{3} T_{1}(t) T_{3}(t)\right)[\phi](x) \\
& \quad=\int_{\mathbb{R}^{3}}\left[\widetilde{M}(t ; \xi)(m(\xi)-\widetilde{m}(t ; \xi))-S^{T} \xi \cdot \widetilde{M}(t ; \xi)\right] \widehat{\phi}(\xi) e^{2 \pi i \xi \cdot e^{-t S} x} d \xi .
\end{aligned}
$$

The symbol within brackets is in fact null for any $\xi \in \mathbb{R}^{3} \backslash\{0\}$ and $t \in \mathbb{R}$, because $-S^{T} \xi \cdot \nabla \widetilde{M}(t ; \xi)=\widetilde{M}(t ; \xi)(\widetilde{m}(t ; \xi)-$ $m(\xi))$. This ends the proof of the lemma.

Proof of Theorem 3.1. The existence of weak/strong solutions in $L^{2}\left(\mathbb{R}^{3}, \mathbb{R}^{3}\right)$ can be proved in several ways: for instance, it is easy to show that $\mathscr{L}$ is a bounded perturbation of the infinitesimal generator of a strongly continuous group on $L^{2}\left(\mathbb{R}^{3}, \mathbb{R}^{3}\right)$, and the existence of solutions follows from classical semigroup theory on Banach space. In this work, we make use instead of the representation formula, by showing that $G(t) \phi_{0}$ defines a weak/strong solution of LSG in $L^{2}\left(\mathbb{R}^{3}, \mathbb{R}^{3}\right)$, depending on the choice of the initial data. We start by showing that the operators $T_{1}(t), T_{2}(t)$ and $T_{3}(t)$ satisfy certain properties, namely

$$
\left\|T_{j}(t) \phi\right\|_{L^{2}} \lesssim_{t}\|\phi\|_{L^{2}} \quad \text { and } \quad\left\|T_{j}(t) \phi-T_{j}(r) \phi\right\|_{L^{2}} \rightarrow 0 \quad \text { as } \quad r \rightarrow t,
$$

for $j=1,2,3$ and $\phi \in L^{2}\left(\mathbb{R}^{3}, \mathbb{R}^{3}\right)$.

For the family of operators $\left\{T_{1}(t)\right\}_{t \in \mathbb{R}}$, we notice that $\left\|T_{1}(t) \phi\right\|_{L^{2}\left(\mathbb{R}^{3}, \mathbb{R}^{3}\right)}=\|\phi\|_{L^{2}\left(\mathbb{R}^{3}, \mathbb{R}^{3}\right)}$ for any $\phi \in L^{2}\left(\mathbb{R}^{3}, \mathbb{R}^{3}\right)$, because $x \mapsto e^{-t S} x$ is a volume-preserving diffeomorphism on $\mathbb{R}^{3}$. We can also prove that

$$
\lim _{r \rightarrow t}\left\|T_{1}(t) \phi-T_{1}(r) \phi\right\|_{L^{2}} \leq \lim _{h \rightarrow 0}\left\|T_{1}(h) \phi-\phi\right\|_{L^{2}}=0,
$$

for all $\phi \in L^{2}\left(\mathbb{R}^{3}, \mathbb{R}^{3}\right)$. In fact, by density of $C_{0}^{\infty}\left(\mathbb{R}^{3}, \mathbb{R}^{3}\right)$ in $L^{2}\left(\mathbb{R}^{3}, \mathbb{R}^{3}\right)$, for any $\phi \in L^{2}\left(\mathbb{R}^{3}, \mathbb{R}^{3}\right)$ and for any $\varepsilon>0$ there exists a function $f \in C_{0}^{\infty}\left(\mathbb{R}^{3}, \mathbb{R}^{3}\right)$ such that $\|\phi-f\|_{L^{2}}<\varepsilon$. Therefore, we have that

$$
\begin{aligned}
\left\|T_{1}(h) \phi-\phi\right\|_{L^{2}} & \leq\left\|T_{1}(h)(\phi-f)\right\|_{L^{2}}+\|f-\phi\|_{L^{2}}+\left\|T_{1}(h) f-f\right\|_{L^{2}} \\
& <2 \varepsilon+\left\|T_{1}(h) f-f\right\|_{L^{2}} .
\end{aligned}
$$

Now, since $f$ is smooth, we can apply the mean value theorem and find a $\delta>0$ such that $\left\|T_{1}(h) f-f\right\|_{L^{2}}<\varepsilon$ if $|h|<\delta$, proving the convergence of the required limit. Regarding the operators $\left\{T_{2}(t)\right\}_{t \in \mathbb{R}}$, we clearly have that $\left\|T_{2}(t) \phi\right\|_{L^{2}} \leq e^{|t||S|}\|\phi\|_{L^{2}\left(\mathbb{R}^{3}, \mathbb{R}^{3}\right)}$ and, as $r \rightarrow t$,

$$
\left\|T_{2}(t) \phi-T_{2}(r) \phi\right\|_{L^{2}\left(\mathbb{R}^{3}, \mathbb{R}^{3}\right)}=\left\|\left(e^{-t S^{T}}-e^{-r S^{T}}\right) \phi\right\|_{L^{2}\left(\mathbb{R}^{3}, \mathbb{R}^{3}\right)} \rightarrow 0 .
$$


Finally, by Plancherel theorem, we have that

$$
\left\|T_{3}(t) \phi\right\|_{L^{2}\left(\mathbb{R}^{3}, \mathbb{R}^{3}\right)} \leq e^{|t|\|m\|_{L^{\infty}\left(\mathbb{R}^{3}\right)}\|\phi\|_{L^{2}\left(\mathbb{R}^{3}, \mathbb{R}^{3}\right)} .}
$$

Since the symbol $\widetilde{M}$ is smooth in $t$, we also have that, as $r \rightarrow t$,

$$
\left\|T_{3}(t) \phi-T_{3}(r) \phi\right\|_{L^{2}\left(\mathbb{R}^{3}, \mathbb{R}^{3}\right)} \leq\|\widetilde{M}(t ; \cdot)-\widetilde{M}(r ; \cdot)\|_{L^{\infty}\left(\mathbb{R}^{3}\right)}\|\phi\|_{L^{2}\left(\mathbb{R}^{3}, \mathbb{R}^{3}\right)} \rightarrow 0 .
$$

By using the estimates above, we have that for any $t \in \mathbb{R}, G(t)$ is a bounded operator from $L^{2}\left(\mathbb{R}^{3}, \mathbb{R}^{3}\right)$ to $L^{2}\left(\mathbb{R}^{3}, \mathbb{R}^{3}\right)$. Moreover, from (3.4) we have that the function $t \mapsto G(t) \phi_{0}=T_{1}(t) T_{2}(t) T_{3}(t) \phi_{0}$ is continuous. We need to prove that $\phi$ satisfies the integral Eq. (1.22), so we look at the following integral: for any test function $f \in C_{C}^{\infty}\left(\mathbb{R}^{3} \times \mathbb{R}, \mathbb{R}^{3}\right)$

$$
\begin{aligned}
\int_{\mathbb{R}} \int_{\mathbb{R}^{3}} G(t) \phi_{0}(x) \cdot\left(\frac{\partial}{\partial t} f(x, t)+\mathscr{K} f(x, t)\right) d x d t \\
=\int_{\mathbb{R}^{3}} \phi_{0}(x) \cdot \int_{\mathbb{R}} G(t)^{\prime}\left(\frac{\partial}{\partial t} f(x, t)+\mathscr{K} f(x, t)\right) d t d x,
\end{aligned}
$$

where $G(t)^{\prime}$ is the operator adjoint to $G(t)$ in $L^{2}\left(\mathbb{R}^{3}, \mathbb{R}^{3}\right)$ for any $t \in \mathbb{R}$, and one can prove that it is given by

$$
G(t)^{\prime}[\phi](x)=\int_{\mathbb{R}^{3}} \bar{M}(t ; \xi) e^{-t S} \widehat{\phi}(\xi) e^{2 \pi i \xi \cdot e^{t S} x} d \xi,
$$

where the symbol $\bar{M}$ is defined in (1.19). From a manipulation of the operator $G(t)^{\prime}$ and Lemma 3.2, it follows that

$$
G(t)^{\prime}\left(\frac{\partial}{\partial t} f(\cdot, t)+\mathscr{K} f(\cdot, t)\right)=\frac{\partial}{\partial t}\left(G(t)^{\prime} f(\cdot, t)\right),
$$

which shows that $G(t) \phi_{0}$ is a weak solution of LSG in $L^{2}\left(\mathbb{R}^{3}, \mathbb{R}^{3}\right)$, as in Definition 1.7.

If $\phi_{0} \in D(\mathscr{L})$, Lemma 3.2 allows us to conclude that $G(t) \phi_{0}$ is a strong solution. In fact, we have that $G(t)(D(\mathscr{L})) \subset D(\mathscr{L})$, and $\frac{d}{d t} G(t) \phi_{0}=\mathscr{L} G(t) \phi_{0} \in L^{2}\left(\mathbb{R}^{3}, \mathbb{R}^{3}\right)$. By continuity of the operators $T_{1}(t), T_{2}(t)$ and $T_{3}(t)$, we infer continuity of the map $t \mapsto \frac{d}{d t} G(t) \phi_{0}=G(t) \mathscr{L} \phi_{0}$, which implies that $G(t) \phi_{0}$ is a strong solution of LSG. The continuous differentiability of the map $t \mapsto G(t) \phi_{0}$ allows us to prove uniqueness by means of an energy estimate and Grönwall inequality.

Finally, we consider a conservative initial datum $\phi_{0}=\nabla f_{0}$, with $f_{0} \in H^{1}\left(\mathbb{R}^{3}\right)$, and the corresponding weak solution $\phi(\cdot, t)=G(t) \nabla f_{0}$. Through a standard computation, one can show that for any smooth $f_{0} \in C_{0}^{\infty}\left(\mathbb{R}^{3}\right)$

$$
\begin{aligned}
G(t) f_{0}(x) & =\nabla \int_{\mathbb{R}^{3}} \widetilde{M}(t ; \xi) \hat{f}_{0}(\xi) e^{2 \pi i \xi \cdot e^{-t S} x} d \xi \\
& =\nabla T_{1}(t) T_{3}(t) f_{0}(x),
\end{aligned}
$$

where we abuse the notation and define $T_{1}(t)$ and $T_{3}(t)$ as operators on scalar valued functions in $L^{2}\left(\mathbb{R}^{3}, \mathbb{R}\right)$ in the natural way. We can conclude the proof by density, by observing that $T_{1}(t) T_{3}(t)$ is a bounded linear operator on $H^{1}\left(\mathbb{R}^{3}\right)$.

\subsection{Existence of Solutions of LSG in $S\left(\mathbb{R}^{3}, \mathbb{R}^{3}\right)$}

We now focus on the existence of solutions of LSG in the Fréchet space $S$ : as we mentioned in the introduction, we endow $S$ with the weak topology generated by the seminorms (1.17). In order to construct strong solutions of LSG in $S$, one way would be to directly apply the theory of locally equicontinuous semigroups on locally convex topological spaces by showing that $L_{1}, L_{2}$ and $L_{3}$ are infinitesimal generators of locally equicontinuous $C_{0}$-groups, and then apply the Trotter formula (see [3, Theorem 20]). Instead, we use the representation formula (1.21) and show that this gives a strong solution in $S$, as by Definition 1.7. In order to do so, we will need estimates for the families of operators $\left\{T_{1}(t)\right\}_{t \in \mathbb{R}},\left\{T_{2}(t)\right\}_{t \in \mathbb{R}}$ and $\left\{T_{3}(t)\right\}_{t \in \mathbb{R}}$, in a similar fashion as for the $L^{2}$-theory.

Theorem 3.3. Given $\phi_{0} \in S$, there exists a unique strong solution $\phi$ in $S$ of $L S G$, as in Definition 1.7. Moreover, the solution is given by $\phi(t)=G(t)\left[\phi_{0}\right]$, as defined in $(1.21)$, and it is smooth, i.e. $\phi \in C^{\infty}(\mathbb{R}, S)$. 
The reader can observe that Schwartz functions belong to $D(\mathscr{L})$, defined in (3.2). Therefore, for any $\phi_{0} \in S$, Theorem 3.1 establishes the existence of a unique strong solution of LSG in $L^{2}\left(\mathbb{R}^{3}, \mathbb{R}^{3}\right)$ corresponding to $\phi_{0}$, and this solution is given by $G(t) \phi_{0}$. We need to establish the regularity of the solution.

Proof of Theorem 3.3. We start by showing that for any $j \in\{1,2,3\}$ and $t \in \mathbb{R}$, the operator $T_{j}(t)$ is bounded in the Fréchet space $S$, in the sense that for any seminorm $p$ on $S$ there exists a seminorm $q$ such that $p\left(T_{j}(t) \phi\right) \lesssim_{t} q(\phi)$ for any $\phi \in S$. We also show that the operators $T_{j}: \mathbb{R} \rightarrow S$ are continuous, i.e. for any semi-norm $p$ on $S$, $p\left(T_{j}(t) \phi-T_{j}(r) \phi\right) \rightarrow 0$ as $r \rightarrow t$, for any $\phi \in S$.

By induction on $|\beta|=\beta_{1}+\beta_{2}+\beta_{3} \in \mathbb{N}$, one can show that for any $\alpha, \beta \in \mathbb{N}^{3}$ there exist constants $C(\alpha)>0$ and $M(\alpha, \beta) \in \mathbb{N}$ and a finite family of seminorms $\left\{\|\cdot\|_{\alpha^{(1)}, \beta^{(1)}}, \ldots,\|\cdot\|_{\alpha^{(M(\alpha, \beta))}, \beta^{(M(\alpha, \beta))}}\right\}$ such that

$$
\left\|T_{1}(t) \phi\right\|_{\alpha, \beta} \leq e^{|t||S|_{\infty}(|\alpha|+|\beta|)} C(\alpha) \sum_{i=1}^{M(\alpha, \beta)}\|\phi\|_{\alpha^{(i)}, \beta^{(i)}},
$$

for any $\phi \in S$ and for any $t \in \mathbb{R}$. Again, by induction on $|\beta|$, one can also show that $\left\|T_{1}(t) \phi-\phi\right\|_{\alpha, \beta} \rightarrow 0$ as $t \rightarrow 0$, for any $\phi \in S$. The operators $T_{2}(t)$ are clearly bounded, as

$$
\left\|T_{2}(t) \phi\right\|_{\alpha, \beta} \leq e^{|t||S|_{\infty}\|\phi\|_{\alpha, \beta},}
$$

for any $\phi \in S$ and $t \in \mathbb{R}$, and the map $t \mapsto T_{2}(t) \phi$ is clearly continuous for all $\phi \in S$.

In order to show that the family of operators $\left\{T_{3}(t)\right\}_{t \in \mathbb{R}}$ also satisfies the wanted properties, the main tool is given by the following estimate for $\mathscr{F}$ and $\mathscr{F}^{-1}$ : for any $\alpha, \beta \in \mathbb{N}^{3}$ we have that

$$
\|\mathscr{F}[\phi]\|_{\alpha, \beta} \leq \sum_{\substack{k \in \mathbb{N}^{3} \\ k \leq \alpha, \beta}} C_{k}^{\alpha, \beta}\left[\|\phi\|_{\beta-k, \alpha-k}+\sum_{i, j=1}^{3}\|\phi\|_{\beta-k+2 e_{i}+2 e_{j}, \alpha-k}\right],
$$

where the constant $C_{k}^{\alpha, \beta}$ is defined as

$$
C_{k}^{\alpha, \beta}:=\sqrt{2} \pi^{2}(2 \pi)^{|\beta-\alpha|} k !\left(\begin{array}{l}
\alpha \\
k
\end{array}\right)\left(\begin{array}{l}
\beta \\
k
\end{array}\right),
$$

and $k \leq \alpha$ for $k, \alpha \in \mathbb{N}^{3}$ means that $k_{i} \leq \alpha_{i}$ for $i \in\{1,2,3\}$. In particular, the following bound

$$
\left\|T_{3}(t) \phi\right\|_{\alpha, \beta} \leq C e^{|t|\|m\|_{L^{\infty}\left(\mathbb{R}^{3}\right)}} \sum_{i=1}^{N}\|\phi\|_{\alpha^{(i)}, \beta^{(i)}},
$$

holds for any $\phi \in S$ and $t \in \mathbb{R}$, with constants $C$ and $N$ depending only on $\alpha, \beta \in \mathbb{N}^{3}$. The continuity of the map $t \mapsto T_{3}(t) \phi$ follows from the fact that the symbol $\widetilde{M}$ is smooth in $t$.

The estimates (3.6), (3.7) and (3.9) prove that the operator $G(t)=T_{1}(t) T_{2}(t) T_{3}(t)$ maps $S$ into $S$, and that for any $\alpha, \beta \in \mathbb{N}^{3}$ there exist constants $C=C(\alpha, \beta, t)$ and $M=M(\alpha, \beta, t)$ and a finite set of multi-indices $\left\{\left(\alpha^{(i)}, \beta^{(i)}\right) \in \mathbb{N}^{3} \times \mathbb{N}^{3}: i \in[1, M] \cap \mathbb{N}\right\}$ such that

$$
\left\|G(t) \phi_{0}\right\|_{\alpha, \beta} \leq C \sum_{i=1}^{M}\left\|\phi_{0}\right\|_{\alpha^{(i)}, \beta^{(i)}},
$$

for any $\phi_{0} \in S$. The continuity of the map $t \mapsto G(t) \phi_{0}$ from $\mathbb{R}$ to $S$ follows from the continuity of the operators $T_{1}(t), T_{2}(t)$ and $T_{3}(t)$ in $t$. By Lemma 3.2, we can write $\frac{d}{d t} G(t) \phi_{0}=G(t)\left[\mathscr{L} \phi_{0}\right]$, and therefore the strong solution is smooth is $t$. A crucial point for the bootstrapping argument is that the operator $\mathscr{L}$ maps $S$ into $S$.

As we mentioned above, the adjoint LSG problem (1.13) is mathematically equivalent to LSG (1.7), therefore the Proof of Theorem 3.3 can be adapted to show the following result. 
Corollary 3.4. Given $\psi_{0} \in S$, there exists a unique strong solution $\psi \in C^{1}(\mathbb{R}, S)$ to the abstract Cauchy problem (1.13). Moreover, the solution is given by $\psi(t)=F(t) \psi_{0}$, where the operator $F(t)$ is defined as

$$
F(t) \psi(x)=\int_{\mathbb{R}^{3}} \bar{M}(t ; \xi) e^{-t S} \widehat{\psi}(\xi) e^{2 \pi i \xi \cdot e^{t S} x} d \xi
$$

for any $\psi \in S$.

The reader can compare the expressions (3.10) and (3.5) and observe that $F(t)=G(t)^{\prime}$, namely $F(t)$ is the adjoint operator of $G(t)$.

\subsection{Existence of Solutions of LSG in $S^{\prime}\left(\mathbb{R}^{3}, \mathbb{R}^{3}\right)$}

The existence of strong solutions of the adjoint LSG (1.13) in $S$ allows us to construct strong solutions to LSG in $S^{\prime}$.

Proof of Theorem 1.2. For any $t \in \mathbb{R}$, we consider the operator $F(t)$ defined in Corollary 3.4, and we prove that $t \mapsto F(t)^{\prime} T_{0}$ is a strong solution of LSG in $S^{\prime}$, in the sense of the Definition 1.7. With $F(t)^{\prime}$ we denote the operator on $S^{\prime}$ defined by duality as

$$
\left\langle F(t)^{\prime} \eta, \psi\right\rangle=\langle\eta, F(t) \psi\rangle, \quad \forall \psi \in S,
$$

for a distribution $\eta \in S^{\prime}$. In fact, the operator $F(t)^{\prime}$ can be seen as an "extension" of the operator $G(t)$ to the space of tempered distributions. In order to prove that $F(t)^{\prime} \eta_{0}$ is a strong solution of LSG, we apply the equivalent of Lemma 3.2 for the operators $\mathscr{K}$ and $F(t)$ : in fact, for any $\eta \in S^{\prime}$ and $t \in \mathbb{R}$, the following sequence of identities

$$
\left\langle\frac{d}{d t} F(t)^{\prime} \eta, \psi\right\rangle=\left\langle\eta, \frac{d}{d t} F(t) \psi\right\rangle=\langle\eta, \mathscr{K} F(t) \psi\rangle=\langle\eta, F(t) \mathscr{K} \psi\rangle=\left\langle\mathscr{L} F(t)^{\prime} \eta, \psi\right\rangle
$$

holds for any test function $\psi \in S$. Continuous differentiability of the function $t \mapsto F(t)^{\prime} \eta_{0}$ from $\mathbb{R}$ to $S^{\prime}$ is a consequence of the continuous differentiability of $t \mapsto F(t) \psi_{0}$ from $\mathbb{R}$ to $S$ for any $\psi_{0} \in S$, as proved in Corollary 3.4.

As we mentioned, $F(t)^{\prime}$ is formally an extension of $G(t)$ to $S^{\prime}$. This is clarified by the following corollary, that connects the $L^{2}$ theory of Theorem 3.1 and the $S^{\prime}$ theory of Theorem 1.2.

Corollary 3.5. Let $\eta_{0}$ be a regular distribution corresponding to the function $\phi_{0} \in L^{2}\left(\mathbb{R}^{3}, \mathbb{R}^{3}\right)$. Then the solution $\eta$ constructed in the Proof of Theorem 1.2 is regular for all times $t \in \mathbb{R}$, i.e. there exists a function $\phi: \mathbb{R} \rightarrow$ $L^{2}\left(\mathbb{R}^{3}, \mathbb{R}^{3}\right)$ such that $\eta(t)=\eta_{\phi(t)}$. The function function $\phi$ is given by the representation formula $\phi(t)=G(t) \phi_{0}$, as defined in (1.21) and therefore it is a weak solution of $L S G$ in $L^{2}\left(\mathbb{R}^{3}, \mathbb{R}^{3}\right)$. If $\phi_{0} \in D(\mathscr{L}) \subset L^{2}\left(\mathbb{R}^{3}, \mathbb{R}^{3}\right)$, then $\phi$ is the unique strong solution of LSG in $L^{2}$.

Proof. The definition of the solution $\eta$ that was constructed in the Proof of Theorem 1.2 and a standard calculation allow us to write for any test function $\psi \in S$ and for any $t \in \mathbb{R}$

$$
\begin{aligned}
\langle\eta(t), \psi\rangle & =\left\langle F(t)^{\prime} \eta_{0}, \psi\right\rangle=\left\langle\eta_{0}, F(t) \psi\right\rangle=\int_{\mathbb{R}^{3}} \phi_{0} \cdot F(t) \psi \\
& =\int_{\mathbb{R}^{3}} G(t) \phi_{0} \cdot \psi=\left\langle\eta_{G(t) \phi_{0}}, \psi\right\rangle .
\end{aligned}
$$

By Theorem 3.1, we have that $G(t) \phi_{0}$ is a weak (strong) solution in $L^{2}\left(\mathbb{R}^{3}, \mathbb{R}^{3}\right)$ of LSG if $\phi_{0} \in L^{2}\left(\mathbb{R}^{3}, \mathbb{R}^{3}\right)$ $\left(\phi_{0} \in D(\mathscr{L})\right)$, and if $\phi_{0}$ is conservative so is $G(t) \phi_{0}$ for all times $t \in \mathbb{R}$ by Theorem 3.1. 


\section{Stability}

As we have an existence theory of a global-in-time solution of LSG, we can study the long-time behaviour of its solutions and comment on stability of the steady solutions introduced in (1.5). A comprehensive theory of stability of such solutions is not the purpose of this work, and we instead consider the particular case of plane-wave perturbations. We begin by characterising the specific forms of the amplitude $a$ and frequency $k$ that give rise to solutions of LSG of the form (1.16), writing their form explicitly in terms of their initial values $a(0)$ and $k(0)$. We then look at their stability, presenting a Proof of Theorem 1.4.

\subsection{Plane-wave Solutions}

We now consider a specific type of solutions to LSG: we are interested in solutions $\phi$ of the form (1.16). As such functions are not square-integrable on $\mathbb{R}^{3}$ for any time $t \in \mathbb{R}$, the concept of plane-wave solutions is clarified in Definition 1.7 (v). It is clear that not all functions of the form (1.16) are plane-wave solutions: indeed, we first characterise the functions $a$ and $k$ that generate plane-wave solutions.

Proposition 4.1. A function $\phi$ of the form in (1.16) is a plane-wave solution of LSG if and only if the functions a and $k$ are of the form

$$
a(t)=a_{0} \widetilde{M}\left(t ; k_{0}\right), \quad k(t)=e^{-t S^{T}} k_{0},
$$

for $a_{0} \in \mathbb{R}$ and $k_{0} \in \mathbb{R}^{3} \backslash\{0\}$, and $\widetilde{M}$ defined in (1.19).

Proof. If $\eta_{\phi}$ is the time-dependent regular distribution corresponding to the function $\phi$ of the form (1.16). Then $\eta_{\phi}$ is a strong solution in $S^{\prime}$ of LSG if and only if for any test function $\psi \in S$ and $t \in \mathbb{R}$ we have that

$$
0=\left\langle\frac{d}{d t} \eta_{\phi(\cdot, t)}, \psi\right\rangle-\left\langle\eta_{\phi(\cdot, t)}, \mathscr{K} \psi\right\rangle=\int_{\mathbb{R}^{3}}\left[\frac{\partial}{\partial t} \phi(x, t)-\mathscr{L} \phi(x, t)\right] \cdot \psi(x) d x,
$$

which holds if and only if $\frac{\partial}{\partial t} \phi-\mathscr{L} \phi=0$. The reader can observe that, although $\mathscr{L} \phi(\cdot, t) \notin L^{p}\left(\mathbb{R}^{3}, \mathbb{R}^{3}\right)$ for any $p \in[1, \infty]$, the product $\mathscr{L} \phi(\cdot, t) \cdot \psi$ is integrable for any Schwartz function $\psi \in S$. From a direct computation one proves that $\frac{\partial}{\partial t} \phi(x, t)=\mathscr{L} \phi(x, t)$ if and only if $a$ and $k$ solve the following initial value problems:

$$
\left\{\begin{array} { l } 
{ a ^ { \prime } ( t ) = m ( k ( t ) ) a ( t ) , } \\
{ a ( 0 ) = a _ { 0 } , }
\end{array} \quad \left\{\begin{array}{l}
k^{\prime}(t)=-S^{T} k(t), \\
k(0)=k_{0} .
\end{array}\right.\right.
$$

The functions in (4.1) are the unique solutions to the Cauchy problems above.

Using the definition of $a$ and $k$ in (4.1), we notice that we can write the solution $\phi$ as

$$
\phi(x, t)=a_{0} \widetilde{M}\left(t ; k_{0}\right) \cos \left(2 \pi k_{0} \cdot e^{-t S} x\right) e^{-t S^{t}} k_{0},
$$

which formally coincides with $G(t)\left[a_{0} k_{0} \cos \left(2 \pi k_{0} \cdot x\right)\right]=G(t)[\phi(\cdot, 0)]$, the representation formula that we found for solutions of LSG in $L^{2}$ and in $S$.

\subsection{Stability of the Plane-wave Solutions}

We proceed now to prove Theorem 1.4, distinguishing between elliptic and hyperbolic flows, as we defined them in (1.12).

Proof of Theorem 1.4. We use different arguments for the two types of flows, so we look at one case at the time. We recall the partition $\operatorname{Sym}_{+}(3, \mathbb{R})=\mathscr{G} \cup \mathscr{B}$ introduced in $(2.7)$. 
I. Hyperbolic flows. For a matrix $A \in \mathscr{A}_{+} \cap \mathscr{G}$, the spectrum of $S$ is $\{0, \lambda,-\lambda\}$, with $\lambda>0$, as we observed in (1.11). We denote with $k_{0}^{ \pm}$an eigenvector of $S^{T}$ corresponding to the eigenvalue $\pm \lambda$, and observe that $\tilde{m}\left(t ; k_{0}^{ \pm}\right)=$ $\pm 2 \lambda$ : in fact,

$$
\begin{aligned}
\pm \lambda & =\frac{S^{T} k_{0}^{ \pm} \cdot A^{-1} k_{0}^{ \pm}}{k_{0}^{ \pm} \cdot A^{-1} k_{0}^{ \pm}} \\
& =\frac{k_{0}^{ \pm} \cdot\left(A^{-1} J-A^{-1} J A^{-1}\right) k_{0}^{ \pm}}{k_{0}^{ \pm} \cdot A^{-1} k_{0}^{ \pm}} \\
& =\frac{e^{\mp t \lambda} k_{0}^{ \pm} \cdot A^{-1} J e^{\mp t \lambda} k_{0}^{ \pm}}{e^{\mp t \lambda} k_{0}^{ \pm} \cdot A^{-1} e^{\mp t \lambda} k_{0}^{ \pm}} \\
& =\frac{e^{-t S^{T}} k_{0}^{ \pm} \cdot A^{-1} J e^{-t S^{T}} k_{0}^{ \pm}}{e^{-t S^{T}} k_{0}^{ \pm} \cdot A^{-1} e^{-t S^{T}} k_{0}^{ \pm}} \\
& =\frac{1}{2} m\left(e^{-t S^{T}} k_{0}^{ \pm}\right)=\frac{1}{2} \widetilde{m}\left(t ; k_{0}^{ \pm}\right) .
\end{aligned}
$$

This allows us to write $\widetilde{M}\left(t ; k_{0}^{ \pm}\right)=e^{ \pm 2 t \lambda}$ and to estimate the $L^{\infty}$-norm of the solution $\phi$ at all times:

$$
\|\phi(\cdot, t)\|_{L^{\infty}\left(\mathbb{R}^{3}, \mathbb{R}^{3}\right)}=\sup _{x \in \mathbb{R}^{3}}|\phi(x, t)|=\left|a_{0}\right| e^{ \pm 2 t \lambda} e^{\mp t \lambda}\left|k_{0}^{ \pm}\right|=\left|a_{0}\right|\left|k_{0}^{ \pm}\right| e^{ \pm t \lambda} .
$$

Hence, if we choose the solution $\phi$ corresponding to an initial datum with $k_{0}$ eigenvector corresponding to the positive eigenvalue, the solution grows exponentially in time. Likewise, if $k_{0}$ is chosen in the eigenspace corresponding to the negative eigenvalue, the solution decays exponentially in time.

If $A \in \mathscr{A}_{+} \cap \mathscr{B}$, then $a$ is constant as $m=0$, and the plane-wave solution $\phi$ is given by

$$
\phi(x, t)=a_{0} \cos \left(2 \pi k_{0} \cdot e^{-t S} x\right) e^{-t S^{T}} k_{0} .
$$

If $k_{0}^{ \pm}$is eigenvector of $S^{T}$ with eigenvalue $\pm \lambda$, then $\|\phi(\cdot, t)\|_{L^{\infty}\left(\mathbb{R}^{3}, \mathbb{R}^{3}\right)}=\left|a_{0}\right| e^{\mp t \lambda}\left|k_{0}\right|$. Therefore the solution with initial datum $\phi_{0}(x)=a_{0} \cos \left(2 \pi k_{0} \cdot x\right) k_{0}$, with $k_{0}$ eigenvector of $S^{T}$ corresponding to the negative eigenvalue, grows exponentially in time in $L^{\infty}$-norm.

II. Elliptic flows. For a generic $A \in \mathscr{A}_{-} \cap \mathscr{G}$, the spectrum of the matrix $S$ is $\{0, i \lambda,-i \lambda\}$, with $\lambda>0$. The trajectories $k(t)=e^{-t S^{T}} k_{0}$ are bounded and periodic with period $\tau=\frac{2 \pi}{\lambda}$, hence the long-term behaviour of the plane-wave solution $\phi$ depends on $\widetilde{M}\left(t ; k_{0}\right)$, as

$$
|\phi(x, t)|=|k(t)|\left|a_{0}\right| \widetilde{M}\left(t ; k_{0}\right)=|k(t)|\left|a_{0}\right| e^{\int_{0}^{t} m(k(r)) d r} .
$$

Since $k(t)$ is $\tau$-periodic, the integral of $m(k(t))$ can be partitioned as below

$$
\int_{0}^{t} m(k(r)) d r=N \int_{0}^{\tau} m(k(r)) d r+\int_{0}^{t-N \tau} m(k(r)) d r,
$$

where $N=\left\lfloor\frac{t}{\tau}\right\rfloor$. The behaviour of the solution depends on the sign of the integral of $m(k(t))$ over the period $[0, \tau]$ : we show that

$$
\int_{0}^{\tau} m(k(r)) d r=0
$$

therefore the integral of $m(k(r))$ over $[0, t]$ is oscillatory, proving that $\phi$ is bounded for any initial data $a_{0}$ and $k_{0}$ :

$$
\|\phi\|_{L^{\infty}\left(\mathbb{R}^{3} \times \mathbb{R}, \mathbb{R}^{3}\right)} \leq \max _{t \in[0, \tau]}|k(t)|\left|a_{0}\right| e^{\tau\|m\|_{L^{\infty}}} .
$$

We need to prove that (4.2) holds for any initial data $a_{0}$ and $k_{0}$. First of all, we observe that we can focus on the numerator of the symbol $m$ defined in (1.9): if $k_{0} \neq 0$, then $k(t) \neq 0$ for all $t$, and there exists a constant $C>1$ such that for all $t \in \mathbb{R}$ 


$$
0<\frac{1}{C} \leq k(t) \cdot A^{-1} k(t) \leq C<\infty .
$$

We use the notation $p(x):=x \cdot A^{-1} J x$ for the numerator of $m$, and we show that there exists a function $f: \mathbb{R} \rightarrow \mathbb{R}$ such that $p(k(t))=f^{\prime}(t)$. Since $\sigma\left(S^{T}\right)=\{0, i \lambda,-i \lambda\}$, there exists an invertible matrix $V \in \mathbb{R}^{3 \times 3}$ such that $S^{T}=-\mu V J V^{-1}$, where $J$ is defined in (1.3), and therefore

$$
\begin{aligned}
p(k(t)) & =k_{0} \cdot e^{-t S} A^{-1} J e^{-t S^{T}} k_{0}=k_{0} \cdot\left(V e^{\mu t J} V^{-1}\right)^{T} A^{-1} J\left(V e^{\mu t J} V^{-1}\right) k_{0} \\
& =k_{0} \cdot V^{-T} e^{-\mu t J} V^{T} A^{-} 1 J V e^{\mu t J} V^{-1} k_{0},
\end{aligned}
$$

where

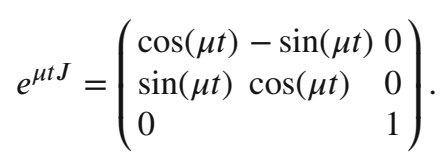

Hence, $p(k(t))=c_{1} \cos ^{2}(\mu t)+c_{2} \cos (\mu t) \sin (\mu t)+c_{3} \cos (\mu t)+c_{4} \sin (\mu t)+c_{5}$, with $c_{1}, \ldots, c_{5} \in \mathbb{R}$ constants depending on $k_{0}$ and $A$. This expression admits an anti-derivative $f(t)$, such that $p(k(t))=f^{\prime}(t)$, given by

$$
f(t)=c_{1} \frac{2 \mu t+\sin (2 \mu t)}{2 \mu}-c_{2} \frac{\cos ^{2}(\mu t)}{2 \mu}+c_{3} \frac{\sin (\mu t)}{\mu}-c_{4} \frac{\cos (\mu t)}{\mu}+c_{5} t+c_{6},
$$

and (4.2) holds if and only if $f(\tau)=f(0)$, namely if and only if $c_{1}+2 c_{5}=0$. This works for any choice of the matrix $A \in \mathscr{A}_{-} \cap \mathscr{G}$.

Finally, if $A \in \mathscr{A}_{-} \cap \mathscr{B}$, then $a$ is constant and $\|\phi(\cdot, t)\|_{L^{\infty}\left(\mathbb{R}^{3}, \mathbb{R}^{3}\right)}=\left|a_{0}\right||k(t)|$ is bounded, concluding the proof.

\section{Comparison with the Quasi-geostrophic Approximation}

We now present a brief comparison between the stability of the family of steady solutions (1.5) which are solutions of both SG and the quasi-geostrophic equations (QG). The two models are obtained as formal asymptotic limits of the 3-D Euler equations for small Rossby number, and the theory presented in this paper can be mirrored for QG following a similar approach. In fact, rather than writing QG in terms of both full and geostrophic velocities (as presented, for instance, in [14]), one can show that the equations can be written in terms of the full velocity $\mathscr{V}[\nabla \phi]$ and the gradient $\nabla \phi$ as follows:

$$
\frac{\partial}{\partial t} \nabla \phi+(J \nabla \phi \cdot \nabla) \nabla \phi+B \mathscr{V}[\nabla \phi]=J \nabla \phi,
$$

where $B=\operatorname{diag}\left(1,1, N^{2}\right), N$ is the Brunt-Väisälä frequency and the operator $\mathscr{V}: \nabla \phi \mapsto u$ is the solution operator associated to the div-curl system

$$
\left\{\begin{array}{l}
\nabla \times(B u)=\nabla \times J \nabla \phi-\nabla \times D^{2} \phi J \nabla \phi, \\
\nabla \cdot u=0,
\end{array}\right.
$$

endowed with decay conditions on the velocity field. The streamfunction $\phi$ is related to the geopotential $P$ in the semi-geostrophic Eq. (1.2) through the identity

$$
P(x, t)=\phi(x, t)+\frac{1}{2} x \cdot B x .
$$

Therefore, the steady solutions $\nabla \bar{\phi}$ for QG corresponding to $\nabla \bar{P}(1.5)$ are given by

$$
\bar{\phi}(x, t)=\frac{1}{2} x \cdot \widetilde{A} x,
$$

with $\widetilde{A}=A-B$ and $A$ a positive definite symmetric matrix. We mention that $\phi$ is not the physical pressure $p$, which is instead given by $p(x, t)=\phi(x, t)+\frac{N^{2}}{2} x_{3}^{2}$, for any $x=\left(x_{1}, x_{2}, x_{3}\right) \in \mathbb{R}^{3}$ and $t \in \mathbb{R}$. 


\subsection{Linearisation of QG}

In a similar fashion as we did in Sect. 2, the quasi-geostrophic Eq. (5.1) can be linearised at a steady solution $\nabla \bar{\phi}$ in (5.2) and written as the following linear problem in the unknown vector field $\psi$

$$
\frac{\partial}{\partial t} \psi+\left(\bar{u}_{g} \cdot \nabla\right) \psi+M^{T} \psi=\mathscr{F}^{-1} \circ \mathscr{M}_{m_{Q G}} \circ \mathscr{F}[\psi],
$$

coupled with the initial condition $\psi(\cdot, 0)=\psi_{0}$. The steady geostrophic velocity $\bar{u}_{g}$ is given by $\bar{u}_{g}(x)=M x$, with the matrix $M$ defined as $M:=J(A-B)$. The symbol $m_{Q G}$ has a similar structure as the symbol $m$ defined in (1.9) for LSG:

$$
m_{Q G}(x):=2 \frac{x \cdot M B^{-1} x}{x \cdot B^{-1} x} .
$$

We refer to the Eq. (5.3) as the linearised quasi-geostrophic equation, or LQG.

By studying the spectrum of the matrix $M$, the reader can show that we can again identify three possible behaviours: we have that $\sigma(M)=\left\{0, \sqrt{\mu_{Q G}},-\sqrt{\mu_{Q G}}\right\}$, with $\mu_{Q G}=b^{2}-(a-1)(d-1)$, where $a, b, c, d, e, f$ are the coefficients of the matrix $A$ as in (1.10). Therefore, the set of positive definite symmetric matrices can be partitioned into the three disjoint sets:

$$
\begin{aligned}
& \mathscr{A}_{+}^{Q G}:=\left\{A \in \operatorname{Sym}_{+}(3): \mu_{Q G}>0\right\}, \\
& \mathscr{A}_{-}^{Q G}:=\left\{A \in \operatorname{Sym}_{+}(3): \mu_{Q G}<0\right\}, \\
& \mathscr{A}_{0}^{Q G}:=\left\{A \in \operatorname{Sym}_{+}(3): \mu_{Q G}=0\right\} .
\end{aligned}
$$

We call hyperbolic and elliptic, respectively, the flows associated to the first and second sets, as we do in the SG theory. In analogy with the work in Sect. 4 , we focus our attention on the two open sets $\mathscr{A}_{+}^{Q G}$ and $\mathscr{A}_{-} Q G$.

It is a straight-forward exercise to prove that the results that we prove for LSG in Sects. 3 and 4 have corresponding analogous for LQG. In particular, we define the operator

$$
\mathscr{L}_{Q G} \psi:=-\left(\bar{u}_{g} \cdot \nabla\right) \psi-M^{T} \psi+\mathscr{F}^{-1} \circ \mathscr{M}_{m_{Q G}} \circ \mathscr{F}[\psi],
$$

and we can derive the representation formula for the solution associated to the initial datum $\psi_{0}$ as

$$
\psi(x, t)=\int_{\mathbb{R}^{3}} e^{-t M^{T}} \widehat{\psi}_{0}(\xi) \widetilde{M}_{Q G}(t ; \xi) e^{2 \pi i \xi \cdot e^{-t M} x} d \xi,
$$

where $\widetilde{M}_{Q G}$ is defined depending on $m_{Q G}$ in (5.4):

$$
\widetilde{m}_{Q G}(t ; \xi)=m_{Q G}\left(e^{-t M^{T}} \xi\right), \quad \widetilde{M}_{Q G}(t ; \xi)=e^{\int_{0}^{t} \widetilde{m}_{Q G}(r ; \xi) d r} .
$$

Theorem 5.1. Given an initial tempered distribution $\eta_{0} \in S^{\prime}$, there exists a strong solution in $S^{\prime}$ of $L Q G(5.3)$, i.e. there exists a $\eta \in C^{1}\left(\mathbb{R}, S^{\prime}\right)$ such that for all $t \in \mathbb{R}$ we have

$$
\left\langle\frac{d}{d t} \eta(t), \psi\right\rangle=\left\langle\mathscr{L}_{Q G} \eta(t), \psi\right\rangle, \quad \forall \psi \in S,
$$

and $\eta(0)=\eta_{0}$ in $S^{\prime}$.

We consider plane-wave solutions of LQG (5.3) of the form (1.16), and consider the corresponding concepts of stability to plane-wave perturbations, as we did in Definition 1.3.

Theorem 5.2. The steady solution $\nabla \bar{\phi}(5.2)$ of $Q G$ (5.1) associated to a matrix $A$ is stable to plane-wave perturbations if it corresponds to an elliptic flow, i.e. $A \in \mathscr{A}_{-}^{Q G}$ as in (5.5), and it is unstable to plane-wave perturbations if it corresponds to an hyperbolic flow, i.e. $A \in \mathscr{A}_{+}^{Q G}$. 


\subsection{Stability in SG and QG}

We showed that the matrix-parameterised family of common steady solutions for SG and QG defined in (1.5) and (5.2) exhibit different behaviours depending on the choice of the positive definite symmetric matrix $A$, and in either approximations we partitioned the set of such matrices into two open subsets (as we already mentioned that the sets $\mathscr{A}_{0}$ and $\mathscr{A}_{0}^{Q G}$ are not interesting). In fact, the two partitions do not coincide, and therefore they allow for four different cases: referring to the sets defined in (1.12) and (5.5), we have that either

(i) $A \in \mathscr{A}_{+} \cap \mathscr{A}_{+}^{Q G}$, or

(ii) $A \in \mathscr{A}_{+} \cap \mathscr{A}_{-}^{Q G}$, or

(iii) $A \in \mathscr{A}_{-} \cap \mathscr{A}_{+}^{Q G}$, or

(iv) $A \in \mathscr{A}_{-} \cap \mathscr{A}_{-}^{Q} G$.

Each of the interections above is non-empty and contains at least one matrix that generates a non-trivial flow. The following matrices are examples of elements in the intersections above, respectively:

$$
\begin{array}{rlrl}
A_{1} & =\left(\begin{array}{lll}
\frac{1}{2} & -1 & -1 \\
-1 & 4 & 1 \\
-1 & 1 & 3
\end{array}\right), & A_{2}=\left(\begin{array}{lll}
2 & 0 & -1 \\
0 & 2 & 0 \\
-1 & 0 & \frac{3}{4}
\end{array}\right), \\
A_{3}=\left(\begin{array}{lll}
\frac{1}{2} & -1 & -1 \\
-1 & 3 & 3 \\
-1 & 3 & \frac{7}{2}
\end{array}\right), & A_{4}=\left(\begin{array}{lll}
\frac{1}{2} & 0 & -1 \\
0 & \frac{1}{2} & 0 \\
-1 & 0 & 3
\end{array}\right) .
\end{array}
$$

The fact that the intersections (ii) and (iii) are non-empty suggests that, although SG and QG are heuristically derived to model the same phenomena, they in fact exhibit different behaviours.

\section{Final Remarks}

We introduced the linearisation of the semi-geostrophic Eq. (1.2) for conservative steady solutions corresponding to globally-defined strictly-convex quadratic geopotentials, and studied the existence of solutions with initial data in $L^{2}\left(\mathbb{R}^{3}, \mathbb{R}^{3}\right), S\left(\mathbb{R}^{3}, \mathbb{R}^{3}\right)$ and $S^{\prime}\left(\mathbb{R}^{3}, \mathbb{R}^{3}\right)$. We discussed the stability of such steady solutions, by introducing the concept of stability to plane-wave perturbations, following the approach in [5]. We also notice that the same techniques can be applied to the well-known quasi-geostrophic equations, and drew a comparison between the long-term behaviour of the same solutions in the two models, LSG (1.1) and LQG (5.3).

We believe that this work is a first step in analysing the dynamical stability of steady solutions to SG (1.2). In particular, it would be interesting to consider more general steady solutions: in Sect. 2, the reader can observe that the derivation makes use of the fact that the Hessian $D^{2} \bar{P}=A$ of the steady geopotential is constant, and lifting this assumption would lead to a different form for the operator $\mathscr{L}$ defined in (1.8). Another issue is to construct non-trivial steady solutions of SG (1.2) both on the whole space $\mathbb{R}^{3}$ and on a domain with boundary: conservative steady solutions with quadratic geopotentials can be constructed if the domain is a ball, but more interesting domains require more complicated solutions, which make it harder to keep calculations as explicit as they are in this paper.

Another point on which the authors would like to draw attention is the concept of stability: our definition of stability to plane-wave perturbations arises from the idea of perturbing the steady solution and studying the longterm behaviour of the perturbation in order to infer something about the behaviour of solutions which are "close" to the steady state. Firstly, we want to remind the reader that a rigorous stability analysis requires a well-posedness theory for the nonlinear Eq. (1.2) that is not available at the moment. Secondly, the concept of stability can be extended to more general perturbations. Again, this can require a more abstract approach, whereas the plane-wave solutions considered in this paper allow for a more explicit investigation of stability. 
Acknowledgements. S. Lisai was supported by The Maxwell Institute Graduate School in Analysis and its Applications (MIGSAA) funded by the UK Engineering and Physical Sciences Research Council (grant EP/L016508/01), the Scottish Funding Council, Heriot-Watt University and the University of Edinburgh. M. Wilkinson was supported by the EPSRC Standard Grant EP/P011543/1. The authors would like to thank Mike Cullen, Beatrice Pelloni and Jacques Vanneste for helpful discussions on this project.

Open Access. This article is licensed under a Creative Commons Attribution 4.0 International License, which permits use, sharing, adaptation, distribution and reproduction in any medium or format, as long as you give appropriate credit to the original author(s) and the source, provide a link to the Creative Commons licence, and indicate if changes were made. The images or other third party material in this article are included in the article's Creative Commons licence, unless indicated otherwise in a credit line to the material. If material is not included in the article's Creative Commons licence and your intended use is not permitted by statutory regulation or exceeds the permitted use, you will need to obtain permission directly from the copyright holder. To view a copy of this licence, visit http://creativecommons.org/licenses/by/4.0/.

Publisher's Note Springer Nature remains neutral with regard to jurisdictional claims in published maps and institutional affiliations.

\section{References}

[1] Ambrosio, L., Colombo, M., De Philippis, G., Figalli, A.: Existence of Eulerian solutions to the semigeostrophic equations in physical space: the 2-dimensional periodic case. Commun. Partial Differ. Equ. 37(12), 2209-2227 (2012)

[2] Ambrosio, L., Colombo, M., De Philippis, G., Figalli, A.: A global existence result for the semigeostrophic equations in three dimensional convex domains. Discrete Continuous Dyn. Syst. - A 34, 1251 (2014)

[3] Albanese, A., Kühnemund, F.: Trotter-Kato approximation theorems for locally equicontinuous semigroups. Rivista di Matematica della Universita di Parma 7(1), 19-53 (2002)

[4] Benamou, J.-D., Brenier, Y.: Weak existence for the semigeostrophic equations formulated as a coupled Monge-Ampère/transport problem. SIAM J. Appl. Math. 58(5), 1450-1461 (1998)

[5] Craik, A.D.D., Criminale, W.O.: Evolution of wavelike disturbances in shear flows?: a class of exact solutions of the navier-stokes equations. Proc. Royal Soc. London. A. Math. Phys. Sci. 406(1830), 13-26 (1986)

[6] Cullen, M.J.P., Kuna, T., Pelloni, B., Wilkinson, M.: Cullen's stability principle and weak solutions of the free-surface semi-geostrophic equations (2018). arXiv preprint arXiv: 1811.03926

[7] Cullen, M.J.P., Shutts, G.J.: Parcel stability and its relation to semigeostrophic theory. J. Atmos. Sci. 44, 1318-1330 (1987)

[8] Cullen, M.J.P.: A Mathematical Theory of Large-Scale Atmosphere/Ocean Flow. Imperial College Press, UK (2006)

[9] Eliassen, A.: The quasi-static equations of motion with pressure as independent variable. Geofis. Publ. 17(3), 5-44 (1948)

[10] Hoskins, B.J.: The geostrophic momentum approximation and the semi-geostrophic equations. J. Atmos. Sci. 32, 233-242 (1975)

[11] Lisai, S., Wilkinson, M.: Smooth solutions of the surface semi-geostrophic equations. Calc. Var. Partial. Differ. Equ. 59, 16 (2020). https:// doi.org/10.1007/s00526-019-1664-3

[12] Pazy, A.: Semigroups of Linear Operators and Applications to Partial Differential Equations, vol. 44. Springer, Berlin (2012)

[13] Ren, S.: Linear stability of the three-dimensional semigeostrophic model in geometric coordinates. J. Atmos. Sci. 55(22), 3392-3402 (1998)

[14] Vallis, G.K.: Geostrophic theory. In: Atmospheric and Oceanic Fluid Dynamics, pp. 171-212. Cambridge University Press (2017)

[15] Yosida, K.: Functional Analysis. Springer, Berlin Heidelberg (1995)

Stefania Lisai

Department of Mathematics, Maxwell Institute for Mathematical

Sciences

Heriot-Watt University

Edinburgh EH14 4AS

UK

e-mail: s137@hw.ac.uk
Mark Wilkinson

Department of Mathematics

Nottingham Trent University

Clifton Campus

Nottingham NG11 8NS

UK

e-mail: mark.wilkinson@ntu.ac.uk

(accepted: March 11, 2021; published online: May 5, 2021) 